\title{
Coupling of multiscale and multi-continuum approaches
}

\author{
Eric T. Chung * Yalchin Efendiev ${ }^{\dagger} \quad$ Tat Leung ${ }^{\ddagger} \quad$ Maria Vasilyeva ${ }^{\S}$ \\ March 16, 2022
}

\begin{abstract}
Simulating complex processes in fractured media requires some type of model reduction. Well-known approaches include multi-continuum techniques, which have been commonly used in approximating subgrid effects for flow and transport in fractured media. Our goal in this paper is to (1) show a relation between multi-continuum approaches and Generalized Multiscale Finite Element Method (GMsFEM) and (2) to discuss coupling these approaches for solving problems in complex multiscale fractured media. The GMsFEM, a systematic approach, constructs multiscale basis functions via local spectral decomposition in pre-computed snapshot spaces. We show that GMsFEM can automatically identify separate fracture networks via local spectral problems. We discuss the relation between these basis functions and continuums in multi-continuum methods. The GMsFEM can automatically detect each continuum and represent the interaction between the continuum and its surrounding (matrix). For problems with simplified fracture networks, we propose a simplified basis construction with the GMsFEM. This simplified approach is effective when the fracture networks are known and have simplified geometries. We show that this approach can achieve a similar result compared to the results using the GMsFEM with spectral basis functions. Further, we discuss the coupling between the GMsFEM and multi-continuum approaches. In this case, many fractures are resolved while for unresolved fractures, we use a multicontinuum approach with local Representative Volume Element (RVE) information. As a result, the method deals with a system of equations on a coarse grid, where each equation represents one of the continua on the fine grid. We present various basis construction mechanisms and numerical results. The GMsFEM framework, in addition, can provide adaptive and online basis functions to improve the accuracy of coarse-grid
\end{abstract}

*Department of Mathematics, The Chinese University of Hong Kong (CUHK), Hong Kong SAR. Email: tschung@math. cuhk. edu.hk. The research of Eric Chung is supported by Hong Kong RGC General Research Fund (Project 400411).

†Department of Mathematics \& Institute for Scientific Computation (ISC), Texas A\&M University, College Station, Texas, USA. Email: efendiev@math.tamu.edu.

${ }^{\ddagger}$ Department of Mathematics, Texas A\&M University, College Station, TX 77843

$\S$ Department of Computational Technologies, Institute of Mathematics and Informatics, North-Eastern Federal University, Yakutsk, Republic of Sakha (Yakutia), Russia, 677980 \& Institute for Scientific Computation, Texas A\&M University, College Station, TX 77843-3368 
simulations. These are discussed in the paper. In addition, we present an example of the application of our approach to shale gas transport in fractured media.

\section{Introduction}

Multiscale phenomena in fractured media. Subsurface formations with discrete fractures, faults, thin features are common in many applications. These include fractured subsurface formations, fractured composite materials, and so on. A main challenge in simulating complex processes is due to multiple scale and high contrast. The material properties within fractures can be very different from the background properties. Due to complex fracture configurations, there are multiple scales and high contrast.

Fine-grid simulation for fractures. Constructing a fine-grid simulation model is typically done in several steps (we refer [21] for the overview). As a first step, an unstructured grid is used to describe the fractures. Then, the flow/transport equations are discretized on the unstructured grid. A variety of techniques have been applied for flow simulation in porous media with discrete fractures using both finite-element and finite-volume methods. Within the finite-element framework, the standard Galerkin formulation ([5, 20, 23, 25]), the mixed finite-element method ([15, 19, 26, 27]), and the discontinuous Galerkin method ([14, 18]) have been used to simulate single-phase and multiphase flow in discrete fracture models. Within the finite-volume framework, formulations have been presented by, e.g., [7, 17, 22, 29, 31, 33]. A hybrid approach combining the finite-element method for the

pressure equation and the finite volume method for transport has also been investigated $([16,28,30])$.

The need for model reduction. Because of multiple scales and high contrast, some type of model reduction is needed for simulating physical processes in fractured media. Typical approaches divide the domain into coarse grids, where effective properties in each coarse-grid block are computed [11, 35]. The standard upscaling methods compute the effective properties using the solution of local problems in each coarse block or representative volume. However, it is known that these approaches are not sufficient as each coarse block contains multiple important modes. This led to multi-continuum approaches [4, 6, 24, 32, 34, 36, where several equations are formulated for each coarse block. In particular, the flow equation for the background (called matrix) and the fracture are written separately with some interaction terms. These approaches make several assumptions such as each continua connected throughout the domain and the form of the coupling. Our goal is to show a relation between these approaches and some multiscale finite element methods and further discuss generalizations based on these approaches.

Brief introduction to the GMsFEM. The GMsFEM [9, 10, 12, follows the framework of the Multiscale Finite Element Method (MsFEM) and is introduced to systematically add new degrees of freedom in each coarse block. The new basis functions are computed by constructing the snapshots and performing local spectral decomposition in the snapshot space. It was shown that there is a spectral gap and the eigenvectors corresponding to very small eigenvalues represent the connected high-conductivity networks. These dominant 
eigenvectors represent fracture networks and can be thought as reduced degrees of freedom representing each continua as explained later.

This paper. In this paper, we discuss a relation between the GMsFEM and the multicontinuum approaches. As mentioned, the dominant eigenvectors represent the connected fracture networks. For example, if there are $n$ separate fracture networks within a coarse block, then we will have $n$ very small eigenvalues and the corresponding eigenvectors represent these fracture networks. We give a detailed comparison between the multi-continua approaches and the GMsFEM in the paper. We discuss the interaction between different continuum media. In multi-continuum approaches, this interaction is modeled based on physical principles, while the GMsFEM approach provides a rigorous coupling between multiple continua. We note the GMsFEM automatically takes into account the interaction of various continua between different coarse blocks, while in multi-continuum approaches, this interaction is stated apriori based on physical principles [37. We also present simplified basis functions that are related to fractures if the fracture networks are identified.

In the paper, we discuss a coupled GMsFEM and the multi-continuum approaches by considering fractures over a very rich hierarchy of scales. Our approach uses multi-continuum at the fine grid and the GMsFEM for modeling the fractures that can be resolved on the fine grid (see Figure 1). In this case, the method deals with a system of equations coupled with the fracture network. First, we discuss a GMsFEM for this system. Secondly, we discuss approaches for computing the parameters of the multi-continuum system based on local Representative Volume Element (RVE) computations. We discuss the setup of local RVE problems and compute the parameters for the multi-continuum fine-grid discretization. Since these parameters, in general, are heterogeneous, the use of coupled basis functions is crucial, and their constructions will be presented. We will also discuss a relation between the GMsFEM and the Multiple Interacting Continua (MINC) 32].

On the other hand, we establish a relation between offline GMsFEM and the multicontinua approaches. The GMsFEM has several important fundamental ingredients that can further be used to achieve higher accuracy and more efficiency. The first ingredient includes the adaptivity. The GMsFEM's adaptivity can be used to add multiscale basis functions in selected regions. This concept can be effectively used to add new multiscale basis functions in selected regions. The second ingredient of the GMsFEM is online basis functions. These basis functions are constructed using the residual information (adaptively in space and time) to speed-up the simulations. This can be used to speed-up the convergence of the proposed method.

We will show numerical results. First, we discuss the GMsFEM's basis construction and numerically show how to identify the number of continua based on local spectral decomposition and the spectrum. Then, we present a simplified basis construction and numerical results for the GMsFEM using both simplified basis construction and a general approach. In the second part, we demonstrate numerical results when the GMsFEM and the multicontinuum approaches are coupled. In this case, the multi-continuum approach is used on the fine grid. Our numerical results use both coupled and un-coupled basis functions and show that the GMsFEM is able to couple with the multi-continuum appraoch and gives 
accurate solution using few basis functions. The GMsFEM can be used for heterogeneously varying multi-continuum problems. In [2], we have applied the GMsFEM to shale gas flows in multi-continuum media. In this paper, we also present an example of the application of our proposed approach to shale gas transport.

Furthermore, we will present an analysis for the GMsFEM when the fine-scale problem is described by a multi-continuum approach. In this case, the method gives a system of coupled equations. We study the convergence of the GMsFEM for cases when basis functions are independently constructed and in a coupled fashion. In both cases, convergence results are obtained.

The paper is organized as follows. In Section 2, we discuss the relation between the GMsFEM and multi-continuum approaches. We develop simplified basis functions and show numerical results. In Section 3, we discuss the coupled GMsFEM and the multi-continuum approach. We also show numerical results in this section. The analysis is given in the Appendix A.

\section{Relation between the GMsFEM and multi-continuum}

The goal of this section is to highlight the similarities between the multi-continuum approaches and the GMsFEM. We assume that the fractures are resolved on a fine grid. We show that (1) the GMsFEM can identify fracture networks and result in a similar system as a multi-continuum approach, (2) the GMsFEM can resolve the detailed fracture and matrix interaction, and (3) the GMsFEM basis functions can be computed in a simplified way.

\subsection{Fine-grid equations in fractured media}

We consider a detailed fine-grid discretization of the flow equation in the fractured media

$$
c \frac{\partial u}{\partial t}=\operatorname{div}(\kappa \nabla u)+q
$$

where $u$ is the solution, $q$ is the source term, $\kappa$ is permeability and $c$ is porosity. The permeability is large within fractures and the porosity has a smaller value in the fractures. Fractures are modeled as one dimensional objects.

The domain $D$ is divided into the fracture and the matrix region

$$
D=D_{m} \oplus_{i} d_{i} D_{f, i}
$$

where $m$ and $f$ represent the matrix and the fracture regions. $d_{i}$ denotes the aperture of the $i$-th fracture and $i$ is the index of the fractures. We denote by $\kappa_{i}$ the permeability of the $i$-th fracture. $D_{m}$ is a two-dimensional domain and $D_{f, i}$ is a one-dimensional domain. The system is written in a finite-element discretization. We introduce the concepts of fine and coarse grids. Let $\mathcal{T}^{H}$ be a coarse-grid partition (computational grid) of the computational domain $D$ into finite elements (triangles, quadrilaterals, tetrahedra, etc.). We assume that 
each coarse element is partitioned into a connected union of fine grid blocks. The fine-grid partition will be denoted by $\mathcal{T}^{h}$, and is by definition a refinement of the coarse grid $\mathcal{T}^{H}$. We use $\left\{x_{i}\right\}_{i=1}^{N}$ (where $N$ denotes the number of coarse nodes) to denote the vertices of the coarse mesh $\mathcal{T}^{H}$ and define the neighborhood of the node $x_{i}$ by

$$
\omega_{i}=\bigcup\left\{K_{j} \in \mathcal{T}^{H} ; \quad x_{i} \in \bar{K}_{j}\right\}
$$

See Figure 1 for illustration.

The bilinear form for the resulting system is

$$
\begin{aligned}
\int_{D_{m}} c_{m} \frac{\partial u_{h}}{\partial t} v_{h} d x & +\sum_{i} \int_{D_{f, i}} c_{f, i} \frac{\partial u_{h}}{\partial t} v_{h} d x \\
& +\int_{D_{m}} \kappa_{m} \nabla u_{h} \cdot \nabla v_{h} d x+\sum_{i} \int_{D_{f, i}} \kappa_{f, i} \nabla_{f} u_{h} \cdot \nabla_{f} v_{h} d x=\int_{D} q v_{h} d x
\end{aligned}
$$

where $v_{h}$ is the fine-grid finite element function, $\nabla_{f}$ is the derivative along the fracture lines, $c_{m}$ and $\kappa_{m}$ porosity and permeability in the matrix, $c_{f, i}$ and $\kappa_{f, i}$ porosity and permeability in the fractures, and $i=1, \ldots, N$. The fracture permeability and porosity include the aperature information $d_{i}$. We remind that in our setup, we assume that a fine grid resolves some set of fractures (very detailed), while each fine grid can contain many small fractures (see Figure 1), i.e., multi-continua.

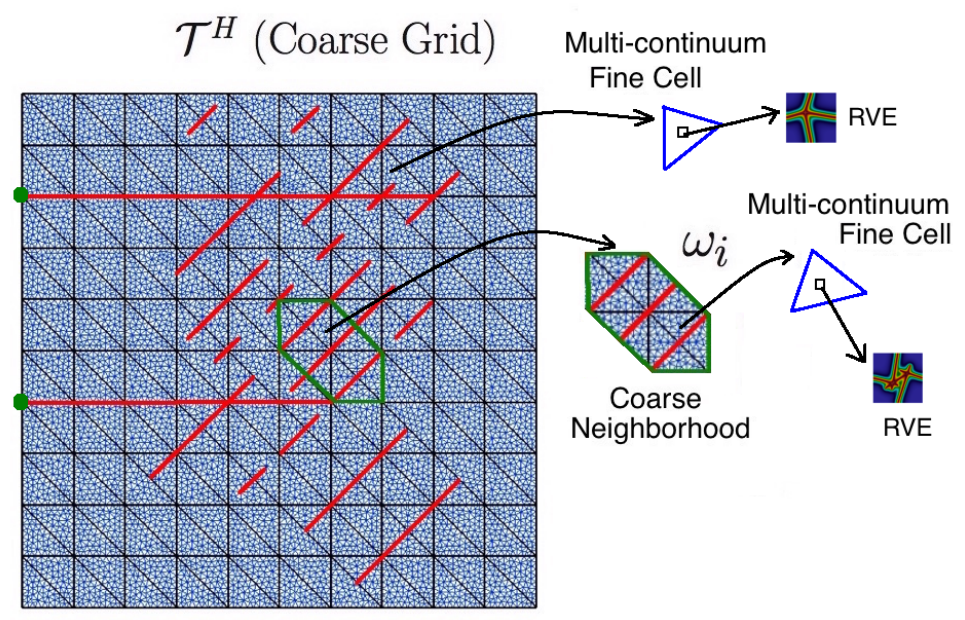

Figure 1: Illustration of a coarse neighborhood and coarse element.

\subsection{Multi-continuum approach. A brief summary.}

The multi-continuum approach is an average model, which is solved on a coarse grid. We denote the solution for $i$-th continuum by $u_{i}$ and assume that each continuum interacts with 
every other, for the sake of generality. Then, we can write the resulting system as

$$
\mathcal{C}_{i i} \frac{\partial u_{i}}{\partial t}=\operatorname{div}\left(\kappa_{i} \nabla u_{i}\right)+Q_{i}\left(u_{1}, \ldots, u_{N}\right)
$$

where $Q_{i}\left(u_{1}, \ldots, u_{N}\right)$ is an exchange term, which can contain both space and time derivates of $u_{i}$ 's [4, 6, 24, 32, 34, 36. In a special case when each continuum only interacts with the background, if the background is $u_{1}$, then

$$
\mathcal{C}_{i i} \frac{\partial u_{i}}{\partial t}=\operatorname{div}\left(\kappa_{i} \nabla u_{i}\right)+Q_{i}\left(u_{1}, u_{i}\right)+q
$$

where $q$ is the source term. We write this equation as

$$
\int_{D} \mathcal{C}_{i i} \frac{\partial u_{i}}{\partial t} v d x+\int_{D} \kappa_{i} \nabla u_{i} \cdot \nabla v d x-\int_{D} Q_{i} v d x=\int_{D} q v d x
$$

where $u_{i}$ is solved on a coarse grid using standard basis functions and $v$ is a standard basis function.

\subsection{A brief overview of the GMsFEM.}

We discuss the use of the GMsFEM on a coarse grid. The GMsFEM uses the coarse grid $\mathcal{T}^{H}$ and constructs a local reduced-order model for each coarse block, by constructing snapshot solutions and extracting basis functions. Snapshots are constructed by solving local problems subject to some boundary conditions. Below, we briefly discuss the snapshot calculations and the basis computations.

\subsubsection{Snapshots and multiscale basis.}

We briefly describe the construction of the snapshot space $V_{\text {snap }}^{\omega_{i}}$. We refer to [9, 12] for further discussions. The snapshot space consists of local solutions. Harmonic functions can be used to construct a snapshot space. We define $\delta_{l}^{h}(x)$, where $\delta_{l}^{h}(x)=\delta_{l, j}, \forall l, j \in J_{h}\left(\omega_{i}\right)$, where $J_{h}\left(\omega_{i}\right)$ denotes the fine-grid boundary node on $\partial \omega_{i}$ and solve the local problems with $\delta_{l}^{h}(x)$ as boundary conditions. More precisely, given a fine-scale piecewise linear function defined

on $\partial \omega$ ( $\omega$ is a coarse block and we omit the index $i)$, we define $\psi_{l}^{\omega, \text { snap }}$ by the following variational problem

$$
a\left(\psi_{l}^{\omega, \text { snap }}, v\right)=\int_{\omega} \kappa_{m} \nabla \psi_{l}^{\omega, \text { snap }} \cdot \nabla v_{h} d x+\sum_{j} \int_{D_{f, j} \cap \omega} \kappa_{f, j} \nabla_{f} \psi_{l}^{\omega, \text { snap }} \cdot \nabla_{f} v_{h} d x=0 \quad \text { in } \omega
$$

and $\psi_{l}^{\omega \text {,snap }}=\delta_{l}^{h}(x)$ on $\partial \omega$. Note that the source is also placed on fracture boundaries. The snapshot space is defined as

$$
V_{\text {snap }}=\operatorname{span}\left\{\psi_{l}^{\text {snap }}: \quad 1 \leq l \leq L_{i}\right\}
$$


where $L_{i}$ is the number of functions in the snapshot space in $\omega$ (a generic coarse block). We also denote

$$
R_{\text {snap }}=\left[\psi_{1}^{\text {snap }}, \ldots, \psi_{L_{i}}^{\text {snap }}\right] .
$$

We note that the randomized boundary conditions [8] can be used to reduce the computational cost. In particular, we solve local problems subject to the boundary condition

$$
\psi_{l}^{\text {snap }}=r_{l},
$$

where $r_{l}$ takes independent random values at every grid block in an oversampled region $\omega^{+}$, $\omega \subset \omega^{+}$(see [8] for details). In this way, we can compute only $n+4$ snapshots for $n$ offline basis vectors.

To construct the offline space, $V_{\text {off }}^{\omega}$, the local spectral problem is solved in the snapshot space [13]. More precisely,

$$
A^{\mathrm{off}} \Psi_{l}^{\mathrm{off}}=\lambda_{l}^{\mathrm{off}} S^{\mathrm{off}} \Psi_{l}^{\mathrm{off}}
$$

where

$$
\begin{gathered}
A^{\text {off }}=\left[a_{m n}^{\text {off }}\right]=\int_{\omega} \kappa_{m} \nabla \psi_{m}^{\text {snap }} \cdot \nabla \psi_{n}^{\text {snap }} d x+\sum_{j} \int_{D_{f, j} \cap \omega} \kappa_{f, j} \nabla_{f} \psi_{m}^{\text {snap }} \cdot \nabla_{f} \psi_{n}^{\text {snap }} d x \\
S^{\text {off }}=\left[s_{m n}^{\text {off }}\right]=\int_{\omega} \kappa_{m} \psi_{m}^{\text {snap }} \psi_{n}^{\text {snap }} d x+\sum_{j} \int_{D_{f, j} \cap \omega} \kappa_{f, j} \psi_{m}^{\text {snap }} \psi_{n}^{\text {snap }} d x .
\end{gathered}
$$

To compute the offline space, we choose $M_{\text {off }}^{\omega}$ smallest eigenvalues and form $\psi_{m}^{\text {off }}=\sum_{l=1}^{L_{i}} \Psi_{m l}^{\text {off }} \psi_{l}^{\text {snap }}$ for $m=1, \ldots, M_{\mathrm{off}}^{\omega}$. Furthermore, the partition of unity functions $\chi_{i}$ (taken to be linear basis functions supported in $\omega_{i}$ ) is multiplied by the eigenfunctions in the offline space $V_{\text {off }}^{\omega_{i}}$ to construct the resulting basis functions

$$
\psi_{i, j}=\chi_{i} \psi_{j}^{\omega_{i}, \text { off }} \quad \text { for } 1 \leq i \leq N \text { and } 1 \leq j \leq M_{\mathrm{off}}^{\omega_{i}}
$$

Here $M_{\text {off }}^{\omega_{i}}$ denotes the number of offline eigenvectors that are selected for each coarse node $i$. With the partition of unity functions, we obtain conforming basis functions in the space

$$
V_{\text {off }}=\operatorname{span}\left\{\psi_{i, j}: 1 \leq i \leq N \text { and } 1 \leq j \leq M_{\text {off }}^{\omega_{i}}\right\}
$$

We can write $V_{\text {off }}=\operatorname{span}\left\{\psi_{i}\right\}_{i=1}^{N_{c}}$, where $N_{c}=\sum_{i=1}^{N} M_{\text {off }}^{\omega_{i}}$ (here, we use a single index) and define

$$
R^{T}=\left[\psi_{1}, \ldots, \psi_{N_{c}}\right] \text {, }
$$

where $\psi_{i}$ are nodal values of each basis function defined on the fine grid.

We remark that there are other discretizations, such as discontinuous Galerkin methods, hybridized Galerkin methods, or other methods. Multiscale basis functions can be constructed following a general framework [9]. The use of discontinuous basis functions coupled within DG can be an attractive approach for these applications and we will study it in the future. 


\subsection{A numerical example demonstrating fracture networks and associated eigenvalues}

Next, we discuss some properties of multiscale basis functions, which show that the GMsFEM basis functions can identify the fracture networks in a general case. Further, we present some simplified basis computations, when the fracture networks have simplistic geometries. We note that each basis function represents a connected fracture network. To show this, we depict an example in Figure 2 with several fractures. In the figure, we also show the eigenvalues. It can be observed that there are three very small eigenvalues and the fourth one is large. The eigenvalue distribution shows that there are three fracture networks. Our construction can detect the fracture networks when fractures have a complex spatial distribution. Moreover, multiscale basis functions can capture the interaction between the fracture and the background media.
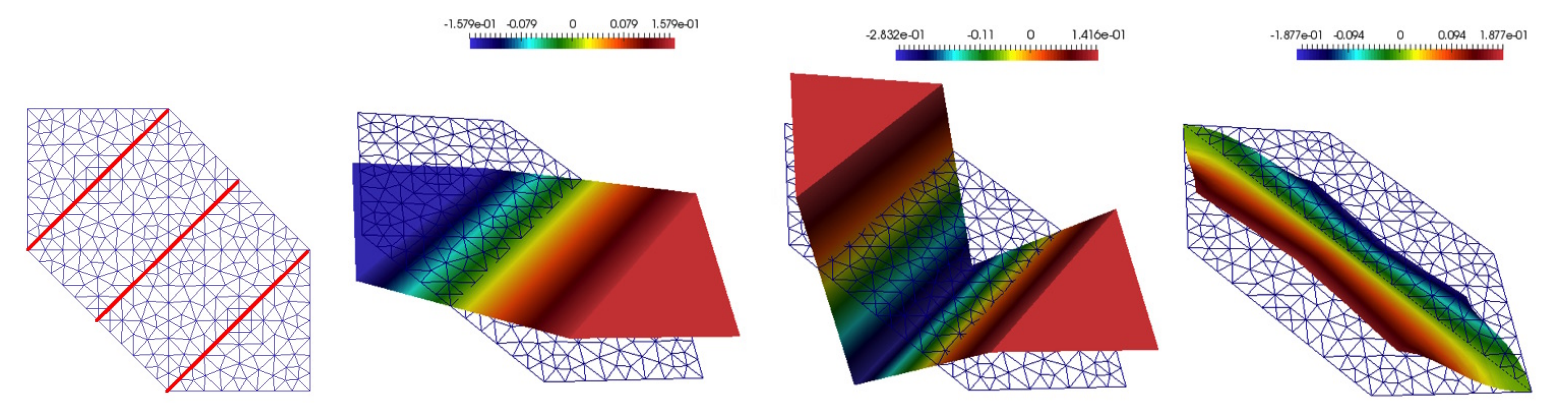

Figure 2: GMsFEM basis functions in a domain $\omega$. The eigenvalues are $\lambda_{1}=1.26 \cdot 10^{-14}$, $\lambda_{2}=2.3 \cdot 10^{-7}, \lambda_{3}=7.0 \cdot 10^{-7}, \lambda_{4}=0.16$.

\subsection{Simplified basis functions.}

For simple cases, simplified basis functions can be constructed. For these basis functions, we can choose constants within fracture networks and solve local problems. In this way, we can avoid a general procedure. Our main approach, which we will test, is the following. For each $\omega$, we define the fracture networks $\Gamma_{1}^{\omega}, \ldots, \Gamma_{M}^{\omega}$ (see Figure 44). Each fracture network intersects with the boundary of $\omega$ at the points $B_{i}^{\Gamma_{j}^{\omega}}$. Then, the multiscale basis functions are defined as

$$
\begin{gathered}
L\left(\phi_{m}\right)=0, \\
\phi_{m}\left(B_{i}^{\Gamma^{\omega}}\right)=\delta_{m j} .
\end{gathered}
$$

Here, $L$ corresponds to the local solution operator (7). These basis functions are multipled by the partition of unity functions. The basis functions are plotted in Figure 3 .

Remark 1. There are other possible approaches that can be considered. For example, the following approach can be an alternative. We denote each rectangle $K \subset \omega$ and denote 

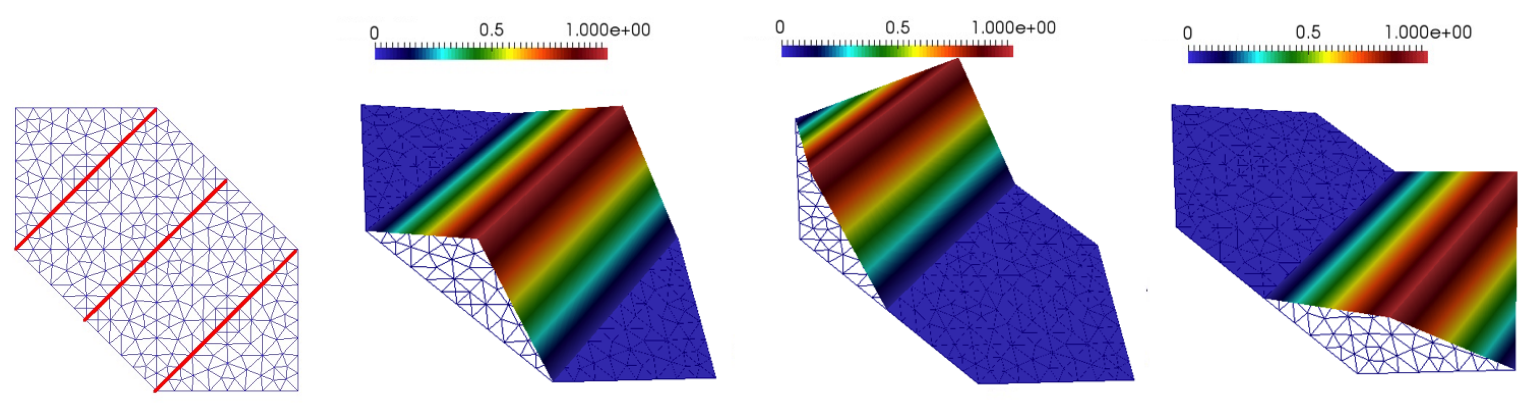

Figure 3: Simplified basis functions in a domain $\omega$.

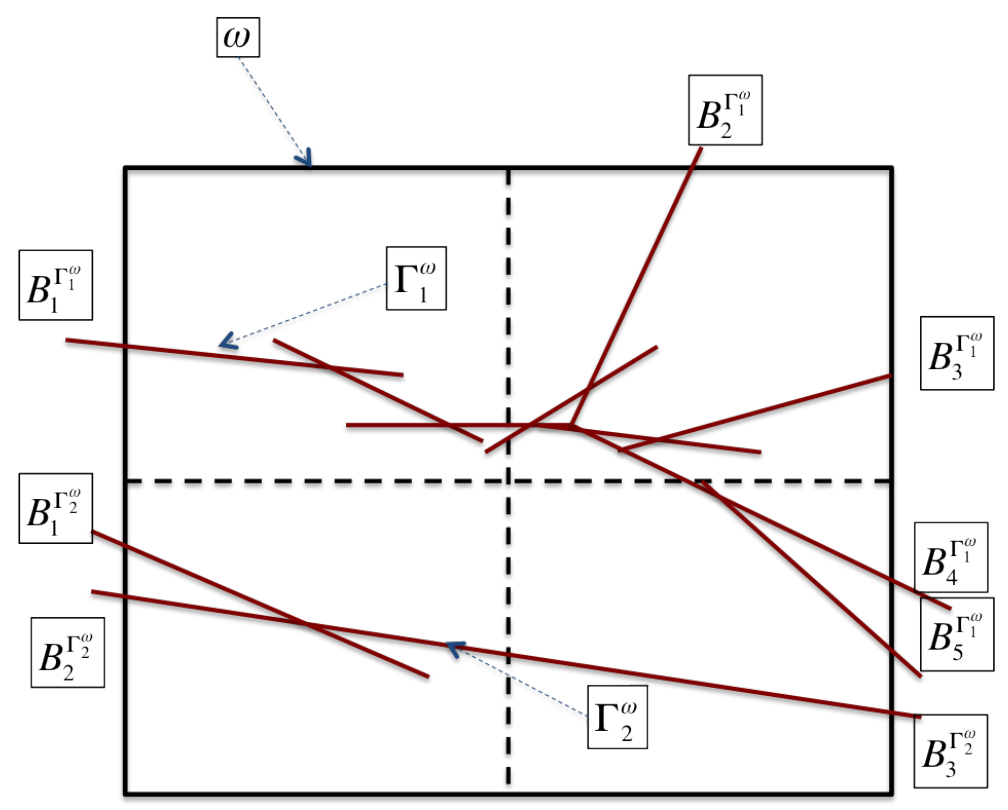

Figure 4: Illustration of coarse neighborhood and simplified basis functions.

internal edges by $\partial \omega^{I}$. We denote the fractures by $\Gamma_{1}^{K}, \ldots, \Gamma_{M}^{K}$ in $K$ and the boundary nodes

$$
B_{i}=B_{i}^{\Gamma_{j}^{K}}
$$

Then, the multiscale basis functions are defined as

$$
\begin{gathered}
L\left(\phi_{m}\right)=0, \\
\phi_{m}\left(B_{i}\right)=\delta_{m i} .
\end{gathered}
$$




\subsection{Relating basis to fractures}

If we denote the basis function for the $i$-th network by $\phi_{i}^{\omega_{j}}$, as described above, then

$$
u_{i}=\sum_{j} c_{i}^{\omega_{j}} \phi_{i}^{\omega_{j}}
$$

Note that

$$
u=\sum_{i} u_{i}
$$

In this case, the coarse-grid equation obtained by the GMsFEM for the basis representing the $i$-th fracture network can be written as

$\int_{D} c \frac{\partial u_{i}}{\partial t} \phi_{i}^{\omega_{l}} d x+\int_{D} \kappa \nabla u_{i} \cdot \nabla \phi_{i}^{\omega_{l}} d x=\int_{D} q \phi_{i}^{\omega_{l}} d x-\sum_{s, s \neq i} \int_{D} c \frac{\partial u_{s}}{\partial t} \phi_{i}^{\omega_{l}} d x-\sum_{s, s \neq i} \int_{D} \kappa \nabla u_{s} \cdot \nabla \phi_{i}^{\omega_{l}} d x$

$l=1, \ldots$ The last two terms represent the interaction of the $i$-th continuum with the other continua. In a special case when the interaction is only with the background, this implies that the support of $\phi_{i}^{\omega_{l}}$ and $\phi_{j}^{\omega_{m}}$ is empty unless $j=1$ or $j=i$. In this case, the equation reduces to

$$
\int_{D} c \frac{\partial u_{i}}{\partial t} \phi_{i}^{\omega_{l}} d x+\int_{D} \kappa \nabla u_{i} \cdot \nabla \phi_{i}^{\omega_{l}} d x=\int_{D} q \phi_{i}^{\omega_{l}} d x-\int_{D} c \frac{\partial u_{1}}{\partial t} \phi_{i}^{\omega_{l}} d x-\int_{D} \kappa \nabla u_{1} \cdot \nabla \phi_{i}^{\omega_{l}} d x .
$$

\subsection{A numerical example}

In this example, we take $D=[0,60]^{2}$ and solve

$$
c \frac{\partial u}{\partial t}-\operatorname{div}(\kappa \nabla u)=0, \quad x \in D
$$

by resolving the fractures with an embedded fracture model on the fine grid (see e.g., [1]). The model for $\kappa$ is shown in Figure 1. We choose initial conditions $u=u_{f}=1$ and, as the boundary conditions, we set $u=0$ at the two points $(0,24)$ and $(0,48)$ and on other boundaries we use zero Neuman boundary conditions. Here, $T_{\max }=300$ is the final time. We set $c_{m}=0.1, \kappa_{m}=10^{-2}$ for the matrix coefficients and $c_{f}=0.01, \kappa_{f}=10^{4}$ for the fracture.

We will compare the results in the weighted $L_{2}^{a}(u)$ norm and weighted $H_{1}^{a}(u)$ semi-norm computed as

$$
\left\|\left.e_{u}\right|_{L_{2}}=\right\| u-\left.u_{h}\right|_{L_{2}} /\left.|| u_{h}\right|_{L_{2}}, \quad\left|e_{u}\right|_{H_{1}}=\left|u-u_{h}\right|_{H_{1}} /\left|u_{h}\right|_{H_{1}},
$$

where $\|u\|_{L_{2}}^{2}=\int_{\Omega} k u^{2} d x,|u|_{H_{1}}^{2}=\int_{\Omega}(k \nabla u, \nabla u) d x, u_{h}$ and $u$ are the fine-scale and coarsescale (multiscale) solutions. In the simulation results, we use $\mathcal{M}$ to denote the number of basis functions per coarse element for $u$ and $D O F$ is the number of degrees of freedom. 
In Figure 5, we show solutions at the final time $T_{\max }$. In Table 1, we present relative errors for GMsFEM and simplified basis functions. The top portion of the table ("Standard GMsFEM") uses multiscale basis functions constructed from the spectral problems and take the equal amount of basis functions in each coarse region. Here, $\mathcal{M}$ refers to the number of basis functions per node. As we observe that if we take 4 basis functions per node, the error is below $5 \%$. In the second portion of the table, we show the results if basis functions are selected based on small eigenvalues. $M_{\lambda}$ refers to the case when we take only very small eigenvalues that represent the fracture networks. In this case, the error is small. $M_{\lambda}-1$ and $M_{\lambda}+1$ refer to the cases when we take one less or one more basis functions in each node. In the bottom portion, we use simplified basis functions. As we observe that the simplified basis captures the networks and provide a small error.
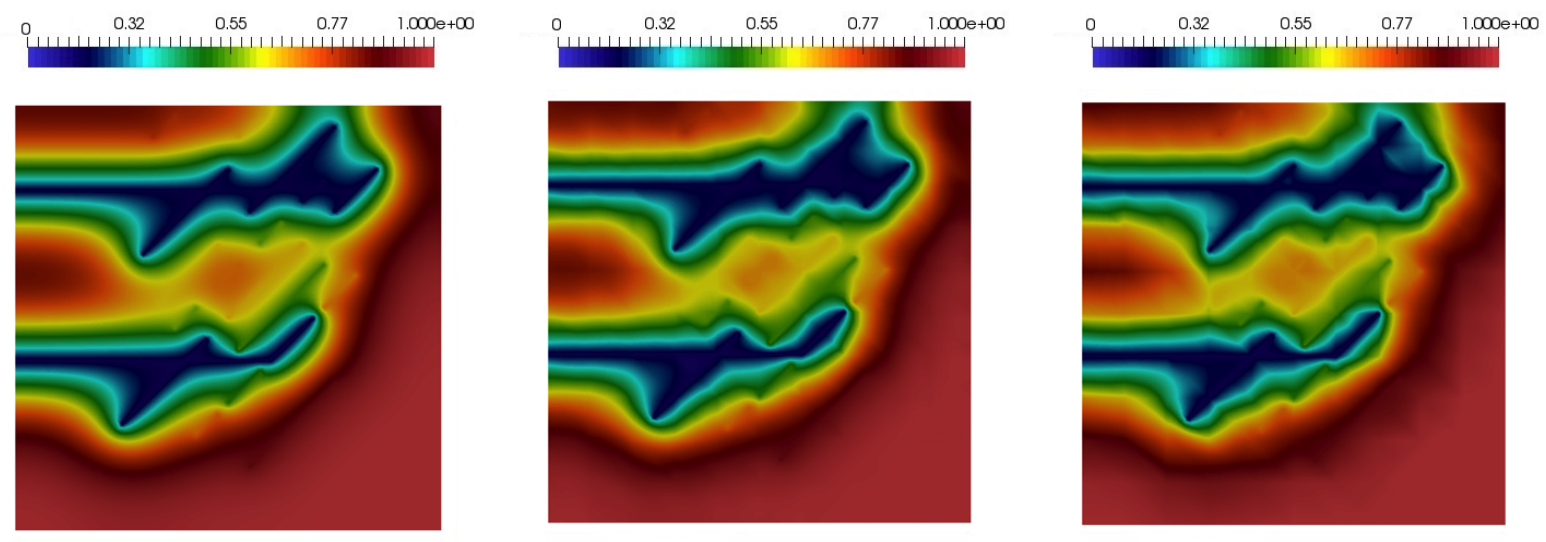

Figure 5: Single-continuum background results. Left: Fine-scale solution $D O F=8917$. Middle: Coarse-scale solution $D O F=396$ using GMsFEM. Right: Coarse-scale solution $D O F=268$ using simplified basis functions.

\section{The coupled GMsFEM and multi-continuum}

In this section, we discuss a combined GMsFEM and multi-continuum method. We assume that some fractures are resolved on the fine grid, while other fractures are represented using a multi-continuum approach at the fine-grid level. As a result, we deal with a system of equations with reaction tensors. We note that each continuum interacts with the resolved fractures and, also, they interact among themselves. We discuss coupled and un-coupled basis constructions. The coupled basis functions are important for some flow scenarios as we discuss. The analysis of the method is given in Appendix A.

We will consider two cases. In the first case, we simply use some values for transfer coefficients $Q$ and in the second case, we compute these transfer coefficients from RVE simulations. In both cases, we assume that each continuum is connected to the fracture. Thus, the fractures are added to each continuum equation with an appropriate weight $\gamma_{i}$, which 


\begin{tabular}{|c|c|c|c|}
\hline $\mathcal{M}$ & $\operatorname{dim}\left(V_{\text {off }}\right)$ & $L_{2}^{a}(u)$ & $H_{1}^{a}(u)$ \\
\hline \multicolumn{4}{|c|}{ Standard GMsFEM } \\
\hline 1 & 121 & 49.067 & 79.885 \\
2 & 242 & 9.116 & 36.499 \\
3 & 363 & 2.325 & 8.099 \\
4 & 484 & 1.413 & 3.919 \\
5 & 605 & 0.883 & 2.658 \\
6 & 726 & 0.708 & 1.795 \\
8 & 968 & 0.253 & 0.348 \\
16 & 1936 & 0.095 & 0.089 \\
\hline \multicolumn{4}{|c|}{ GMsFEM by $\lambda$} \\
\hline$M_{\lambda}-1$ & 184 & 13.554 & 45.342 \\
$M_{\lambda}$ & 275 & 2.651 & 11.377 \\
$M_{\lambda}+1$ & 396 & 1.582 & 4.414 \\
\hline \multicolumn{4}{|c|}{ Simplified basis functions } \\
\hline all & 268 & 1.850 & 3.799 \\
\hline \multicolumn{4}{|c}{} \\
\hline
\end{tabular}

Table 1: Single-continuum background. Numerical results of relative errors (\%) at the final simulation time. $D O F_{f}=8917$.

represents the amount of the fluid passed to the fracture network from the $i$-th continuum. We can assume $\sum_{i} \gamma_{i}=1$. The resulting equations have the following variational form

$$
\begin{aligned}
& \int_{D_{m}} c_{m, s} \frac{\partial u_{s}}{\partial t} v d x+\sum_{i} \int_{D_{f, i}} c_{i, s} \frac{\partial u_{s}}{\partial t} v d x \\
& \quad+\int_{D_{m}} \kappa_{s} \nabla u_{s} \cdot \nabla v d x+\sum_{i} \int_{D_{f, i}} \kappa_{i, s} \nabla u_{s} \cdot \nabla v d x=\int_{D} Q_{s} v d x,
\end{aligned}
$$

$s=1, \ldots, N$. Here, $\kappa_{i, s}$ is the fracture permeability that takes into account the interaction of the $s$-th continuum with the resolved fracture network and $c_{i, s}$ is the mass exchange term that take into account the interaction between the fracture and the $s$-th continuum. Note that $c_{i, s}, \kappa_{i, s}$, and $Q_{s}$ depend on $\gamma_{s}$. This is a coupled system of differential equations with multiscale high-contrast coefficients. The coupling is done via the right hand side and, thus, multiscale basis functions can be constructed for separately for each equation using the high-contrast permeabilities or jointly.

In our numerical simulations, we will consider two approaches for constructing multiscale spaces as described in Appendix A. In the first approach (called un-coupled), multiscale basis functions will be constructed for each continuum separately by considering only the permeability $\kappa_{i}$ and ignoring the transfer functions. This is the same as using single-phase flow basis functions for each continuum and follows the GMsFEM approach discussed above. In the second approach, the multiscale basis functions will be constructed by solving a 
coupled problem for snapshot spaces and performing a spectral decomposition as discussed in Appendix A. The resulting GMsFEM procedure is the same as the one presented in Section 2.3, except that the construction of the snapshot functions is replaced by the coupled approach discussed above. Note that a different spectral problem is used for a coupled basis construction.

We present numerical results. We consider the model shown in Figure 1. We consider a dual porosity system for un-resolved fractures $\left(u_{f}\right)$ and matrix flow $\left(u_{m}\right)$. For the un-resolved fractures parameters, we set $\kappa_{f}=10^{-3}$ and $c_{f}=0.1$. For the matrix parameters, we use $\kappa_{m}=10^{-7}$ and $c_{m}=0.01$. Using DFN (discrete fracture network), we implement a resolved fracture network $\left(u_{F}\right)$, which interacts with both the un-resolved fracture system $(80 \%)$ and the matrix system $(20 \%)$. That is both matrix and un-resolved fracture system communicate with the resolved fractures. We set $\kappa_{F}=10^{3}, c_{F}=0.1$. Here, we use the transfer parameter $Q=250 \cdot \kappa_{m}$ and $T_{\max }=5000$. In Figure 6, we show solutions at the final time. In Table 2. we present relative errors for GMsFEM and simplified basis functions. In this table, we present the results when the basis is computed in a coupled way and separately for each continuum using the flow equation (without transfer functions). From this table, we observe that the GMsFEM using coupled basis functions provides better accuracy compared to that computed with un-coupled basis functions. Moreover, we observe that when choosing 6 basis functions per coarse node, we can obtain an excellent result using the GMsFEM. We have also tested the GMsFEM with simplified basis functions. The results are similar to those obtained from above. In particular, we observe a similar accuracy if we choose only basis functions corresponding to very small eigenvalues. We observe large errors if we do not choose eigenvectors corresponding to very small eigenvalues.
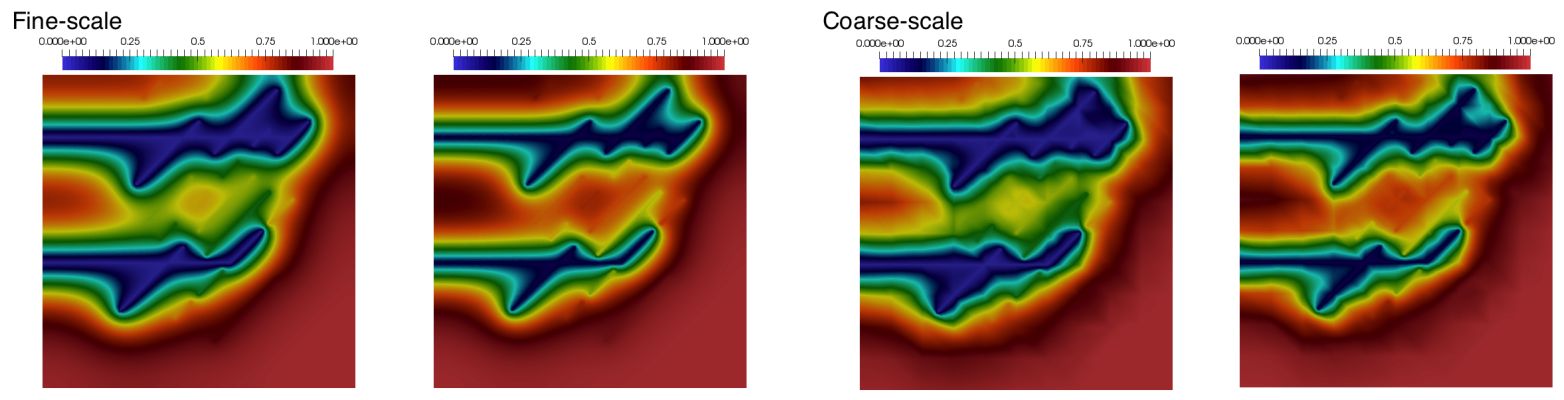

Figure 6: Dual-continuum background. Left: Fine-scale solution $D O F=17834$. Right: Coarse-scale solution $D O F=536$ using simplified basis functions.

\subsection{RVE-based multi-continuum computations}

A representative volume element can be used to compute the parameters in multi-continuum equations. We follow a known procedure (see e.g., [21]). In this approach, we compute the transfer parameters based on RVE simulations. We consider a case of two-continua at the 


\begin{tabular}{|c|c|c|c|c|c|c|}
\hline $\mathcal{M}$ & $\operatorname{dim}\left(V_{\text {off }}\right)$ & $L_{2}^{a}\left(c_{1}\right)$ & $H_{1}^{a}\left(c_{1}\right)$ & $L_{2}^{a}\left(c_{2}\right)$ & $H_{1}^{a}\left(c_{2}\right)$ & $H^{Q}\left(c_{1}, c_{2}\right)$ \\
\hline \multicolumn{7}{|c|}{ Standard GMsFEM (un-coupled) } \\
\hline 6 & 1452 & 0.846 & 1.966 & 0.846 & 15.938 & 4.519 \\
8 & 1936 & 0.297 & 0.379 & 0.297 & 15.835 & 4.093 \\
12 & 2904 & 0.148 & 0.188 & 0.148 & 14.213 & 3.670 \\
16 & 3872 & 0.106 & 0.087 & 0.106 & 11.769 & 3.041 \\
\hline \multicolumn{7}{|c|}{ Simplified basis functions (un-coupled) } \\
\hline all & 536 & 2.386 & 3.831 & 2.386 & 21.765 & 6.713 \\
\hline \multicolumn{7}{|c|}{ Standard GMsFEM (coupled) } \\
\hline 6 & 1452 & 1.919 & 2.767 & 1.919 & 9.919 & 3.588 \\
8 & 1936 & 1.052 & 1.154 & 1.052 & 2.840 & 1.335 \\
12 & 2904 & 0.354 & 0.550 & 0.354 & 1.325 & 0.628 \\
16 & 3872 & 0.124 & 0.102 & 0.124 & 0.643 & 0.194 \\
\hline \multicolumn{7}{|c|}{ Simplified basis functions (coupled) } \\
\hline all & 830 & 2.056 & 3.439 & 2.056 & 6.234 & 3.690 \\
\hline
\end{tabular}

Table 2: Dual-continuum background. Numerical results of relative errors (\%) at the final simulation time. $D O F_{f}=17834 . Q=250 \cdot \kappa_{m}$.

microscale and compute the transfer function based on RVE simulations. For this reason, we solve the local problem with DFN (corresponding to (7))

$$
c \frac{\partial \xi}{\partial t}-\operatorname{div}(\kappa \nabla \xi)=0 \text { in the RVE }
$$

and impose $\xi=1$ at the fracture nodes. One can also use a source term in the fracture or the local eigenvalue problems see [21]. It is assumed that zero Neumann boundary conditions are imposed on the rest of the boundaries. Then, the transfer coefficient is defined as

$$
Q(t)=F_{\text {frac }}(t) /\left(1-\langle\xi\rangle_{\text {matrix }}(t)\right)
$$

$Q(t)$ will quickly reach an asymptote, which is used as a transfer coefficient. Here $\langle\xi\rangle_{\text {matrix }}$ is the volume average over the matrix region.

We present results for a dual-continuum coupled with the GMsFEM. We set $c_{f}=c_{m}=$ $0.1, \kappa_{f}=10^{-3}, \kappa_{m}=10^{-7}$, and $c_{F}=0.01, \kappa_{F}=10^{3}$ for the fracture. Using DFN, we implement the resolved fracture network with $80 \%$ in $c_{f}$ and $20 \%$ to $c_{m}$ as well as $T_{\max }=$ 5000. Here, we use the transfer functions $Q_{1}=500 \cdot \kappa_{m}$ for $y<5 L_{y} / 10, Q_{2}=920 \cdot \kappa_{m}$ for $y>7 L_{y} / 10$, and linearize $Q$ in between them, i.e., $\left.y \in 5 L_{y} / 10,7 L_{y} / 10\right)$. The values of $Q$ are computed using local RVE simulations. In particular, we set the pressure to be one and compute $Q$ as the flux over the pressure difference in the fracture and the average pressure in the matrix (see [21]).

In Figure 7, we plot both the fine-scale and the coarse-scale solutions at the final time, and in Table 3, we report the relative errors for GMsFEM and simplified basis functions. The 
numerical results are obtained using the parameters as follows: $c_{f}=c_{m}=0.1, \kappa_{f}=10^{-3}$, $\kappa_{m}=10^{-7}$ and $c_{F}=0.01, \kappa_{F}=10^{3}$ for the fracture. The resolved fracture network is implemented using DFN with $80 \%$ in $c_{f}$ and $20 \%$ to $c_{m}$. In addition, we take the transfer function $Q=250 \cdot \kappa_{m}$ and $T_{\max }=5000$. The final time solution plots are shown Figure 7 , and the corresponding relative errors are reported in Table 3, where the results are shown when the basis are computed in a coupled way and separately for each continuum using flow equation (without transfer functions). Based on these results, we conclude that the GMsFEM using coupled basis functions provides better accuracy compared to that computed with uncoupled basis functions. Furthermore, we observe that when choosing 6 basis functions per coarse node, we can obtain an excellent result using the GMsFEM. On the other hand, we tested the performance of our method using simplified basis functions, and observed a similar accuracy. Finally, we observe large errors if we do not choose eigenvectors corresponding to very small eigenvalues.
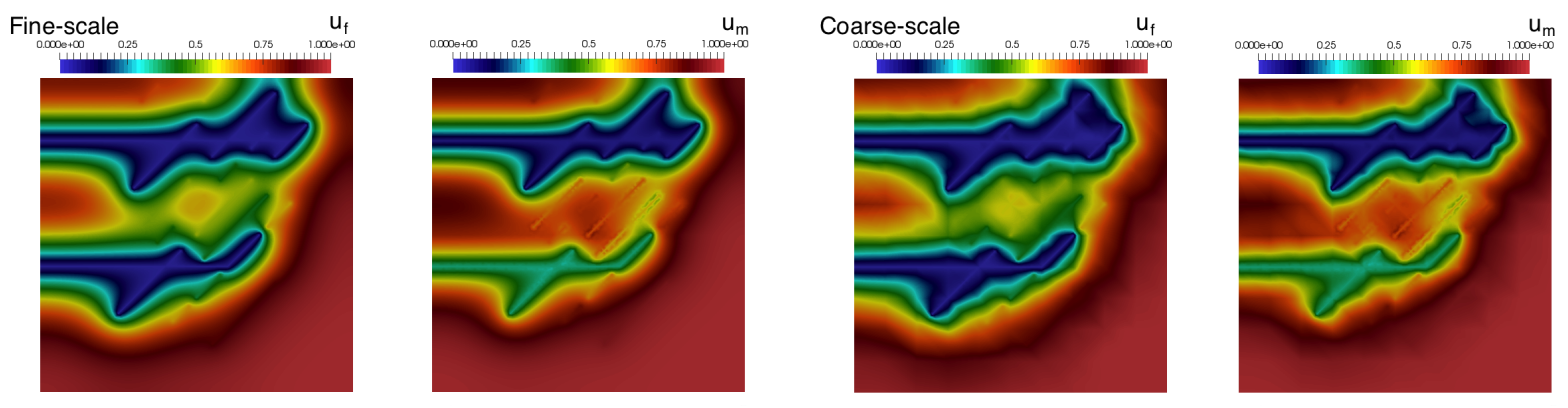

Figure 7: Dual-continuum background with $Q=Q(x)$. Left: Fine-scale solution $D O F=$ 17834. Right: Coarse-scale solution $D O F=536$ using simplified basis functions. $Q_{1}=$ $250 \cdot \kappa_{m}$ and $Q_{2}=920 \cdot \kappa_{m}$.

Remark 2. We remark that the RVE can be used to approximate the effective properties. To show this example, we assume that in each fine-grid, the multi-continua can be resolved. In this case, we construct multiscale basis functions via local spectral decomposition in the form

$$
\phi_{i, f i n e}^{\omega_{j}}=\chi_{f i n e}^{\omega_{i}} \psi_{j, f i n e}
$$

As we discussed above, this equation is a multi-continua model obtained via the GMsFEM and can be related to multi-continua (5) by constructing the basis for the corresponding continua.

When using RVEs, the main challenge is to define

$$
\int_{D} \kappa \nabla \phi_{i, f i n e}^{\omega_{j}} \cdot \nabla \phi_{m, f i n e}^{\omega_{l}} d x
$$

using RVE computations. This is based on a localization assumption, which we introduce next. 


\begin{tabular}{|c|c|c|c|c|c|c|}
\hline $\mathcal{M}$ & $\operatorname{dim}\left(V_{\text {off }}\right)$ & $L_{2}^{a}\left(c_{1}\right)$ & $H_{1}^{a}\left(c_{1}\right)$ & $L_{2}^{a}\left(c_{2}\right)$ & $H_{1}^{a}\left(c_{2}\right)$ & $H^{Q}\left(c_{1}, c_{2}\right)$ \\
\hline \multicolumn{7}{|c|}{ Standard GmsFEM (un-coupled) } \\
\hline 6 & 1452 & 0.837 & 1.925 & 0.837 & 30.542 & 13.869 \\
8 & 1936 & 0.293 & 0.358 & 0.293 & 26.427 & 11.912 \\
12 & 2904 & 0.148 & 0.193 & 0.148 & 23.165 & 10.440 \\
16 & 3872 & 0.108 & 0.089 & 0.108 & 17.945 & 8.086 \\
\hline \multicolumn{7}{|c|}{ Simplified basis functions (un-coupled) } \\
\hline all & 536 & 2.343 & 3.870 & 2.343 & 36.649 & 16.872 \\
\hline \multicolumn{7}{|c|}{ Standard GmsFEM (coupled) } \\
\hline 6 & 1452 & 1.944 & 2.584 & 1.944 & 6.942 & 3.934 \\
8 & 1936 & 1.070 & 1.200 & 1.070 & 2.197 & 1.452 \\
12 & 2904 & 0.359 & 0.544 & 0.359 & 0.788 & 0.606 \\
16 & 3872 & 0.129 & 0.105 & 0.129 & 0.375 & 0.193 \\
\hline \multicolumn{7}{|c|}{ Simplified basis functions (coupled) } \\
\hline all & 830 & 2.105 & 3.399 & 2.105 & 4.122 & 3.557 \\
\hline
\end{tabular}

Table 3: Dual-continuum background. Numerical results of relative errors (\%) at the final simulation time. $D O F_{f}=17834 . Q_{1}=250 \cdot \kappa_{m}$ and $Q_{2}=920 \cdot \kappa_{m}$.

We consider $\mathcal{H}^{\omega}$, which is the harmonic expansion in $\omega$, which is defined by solving local problems in each $K$. We can use

$$
\int_{D} \kappa \nabla \mathcal{H}^{\omega_{j}}\left(\phi_{i, f i n e}^{\omega_{j}}\right) \cdot \nabla \mathcal{H}^{\omega_{l}}\left(\phi_{m, \text { fine }}^{\omega_{l}}\right) d x
$$

to approximate the elements of the stiffness matrix. Our localization assumption uses the local snapshots computed in the RVE for each $\omega_{i}$, which we denote by RVE $E_{i}$ We denote these $R V E$ snapshots by $\psi_{j, \text { fine }}^{R V E_{i}}$. Then, we propose the following localization assumption

$$
\int_{D} \kappa \nabla \mathcal{H}^{\omega_{j}}\left(\phi_{i, f i n e}^{\omega_{j}}\right) \cdot \nabla \mathcal{H}^{\omega_{l}}\left(\phi_{m, \text { fine }}^{\omega_{l}}\right) d x \approx \int_{D} \kappa \nabla \mathcal{H}^{R V E_{j}}\left(\chi_{\text {fine }}^{\omega_{j}} \psi_{i, \text { fine }}^{R V E_{j}}\right) \cdot \nabla \mathcal{H}^{R V E_{l}}\left(\chi_{\text {fine }}^{\omega_{l}} \psi_{m, f i n e}^{R V E_{l}}\right) d x
$$

\subsection{Numerical simulation of the shale gas transport}

In this section, we add a case study for our method. We follow the example considered in [2], where a shale gas transport with dual-continuum (organic and inorganic pores) (see also [3]) is studied. In inorganic matter, we have

$$
\varphi_{i} \frac{\partial c}{\partial t}=\operatorname{div}\left(\left(\varphi_{i} D_{i}+c Z R T \frac{\kappa_{i}}{\mu}\right) \nabla c\right)+Q_{k i} .
$$

where $\varphi_{i}$ is the inorganic porosity, $D_{i}$ is the tortuosity corrected coefficient of diffusive molecular transport in the inorganic matrix, $\kappa_{i}$ is the inorganic matrix absolute permeability, 
$\mu$ is the dynamic gas viscosity, $p_{i}$ is the inorganic matrix pressure, $p=c Z R T$ and $Q_{k i}$ is the transfer function.

Here, we use the following transfer function

$$
Q_{k i}=\tau_{k i} \sigma\left(c_{k}-c\right), \quad \tau_{k i}=\varphi_{k} D_{k}+\left(1-\varphi_{k}\right) D_{s} F^{\prime} .
$$

where $\sigma$ is a shape factor.

For free and adsorbed gas in the kerogen $\left(c_{k}\right.$ and $\left.c_{\mu}\right)$

$$
\left(\varphi_{k}+\left(1-\varphi_{k}\right) F^{\prime}\right) \frac{\partial c_{k}}{\partial t}=\operatorname{div}\left(\left(\varphi_{k} D_{k}+\left(1-\varphi_{k}\right) D_{s} F^{\prime}+c_{k} Z R T \frac{\kappa_{k}}{\mu}\right) \nabla c_{k}\right)-Q_{k i}
$$

where $\varphi_{k}$ is the kerogen porosity, $D_{k}$ is the tortuosity corrected coefficient of diffusive molecular transport for the free gas in kerogen, $D_{s}$ is the coefficient of diffusive molecular transport for the adsorbed gas in kerogen, $\kappa_{k}$ is the kerogen permeability, and $p_{k}$ is the kerogen pressure. For $c_{\mu}$ we use linear Henry's isotherm $c_{\mu}=F\left(c_{k}\right), F\left(c_{k}\right)=k_{H} c_{k}$.

For free-gas in fracture network, we have

$$
\varphi_{f} \frac{\partial c_{f}}{\partial t}=\operatorname{div}\left(c_{f} Z R T \frac{\kappa_{f}}{\mu} \nabla c_{f}\right)
$$

where $\varphi_{f}$ is the fracture porosity, $K_{L}$ is the diffusion coefficient, $\kappa_{f}$ is the fracture absolute permeability, and $p_{f}$ is the fracture pressure.

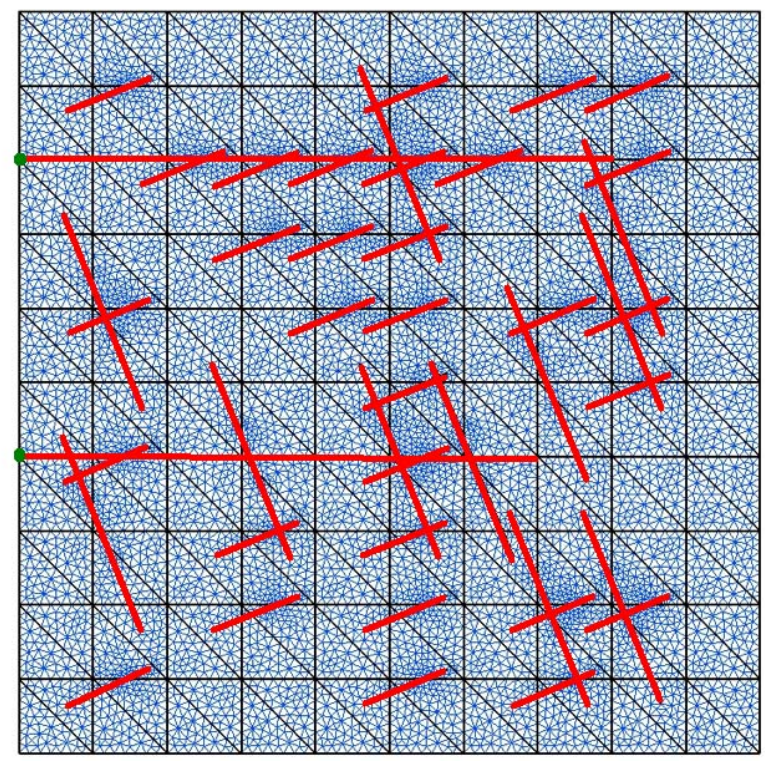

Figure 8: Computational mesh with fractures for shale gas transport.

We consider the model geometry with discrete fracture distribution as shown in Figure 8. The coarse grid is uniform and contains 121 vertices and 200 coarse cells. The domain 
$D$ has a length of 50 meters in both directions. The other model parameters used are as follows. $\quad R=8.31[\mathrm{~J} /(\mathrm{K} \cdot \mathrm{mol})], T=323.0[\mathrm{~K}], Z=1.0, p_{i}=20 \cdot 10^{6}[\mathrm{~Pa}], p_{\text {well }}=5$. $10^{6}[\mathrm{~Pa}], p_{L}=10^{6}[\mathrm{~Pa}], c_{\text {init }}=p_{i} /(Z R T)\left[\mathrm{mol} / \mathrm{m}^{3}\right], c_{\text {well }}=p_{\text {well }} /(Z R T)\left[\mathrm{mol} / \mathrm{m}^{3}\right], \varphi_{i}=0.025$, $\varphi_{k}=0.025, \varphi_{f}=0.01 \kappa_{i}=10^{-19}\left[\mathrm{~m}^{2}\right], \kappa_{k}=0.0\left[\mathrm{~m}^{2}\right], \kappa_{n f}=10^{-14}\left[\mathrm{~m}^{2}\right], \kappa_{h f}=10^{-13}\left[\mathrm{~m}^{2}\right]$, $D_{s}=D_{i}=D_{k}=10^{-8}\left[\mathrm{~m}^{2} / \mathrm{s}\right], k_{H}=0.1, \mu=10^{-5}[\mathrm{~Pa} \cdot \mathrm{s}]$. For transfer functions, we set $\sigma=10.0\left[1 / \mathrm{m}^{2}\right]$.

As we remarked, the purpose of this example is to show the geo-application of our approach. In Figure 9, we depict the solutions at the final time $T_{\max }=500$ days. We observe that the GMsFEM with simplified basis functions provides a good agreement. In this case, we have observed less than $1 \%$ in $L^{2}$ norm.
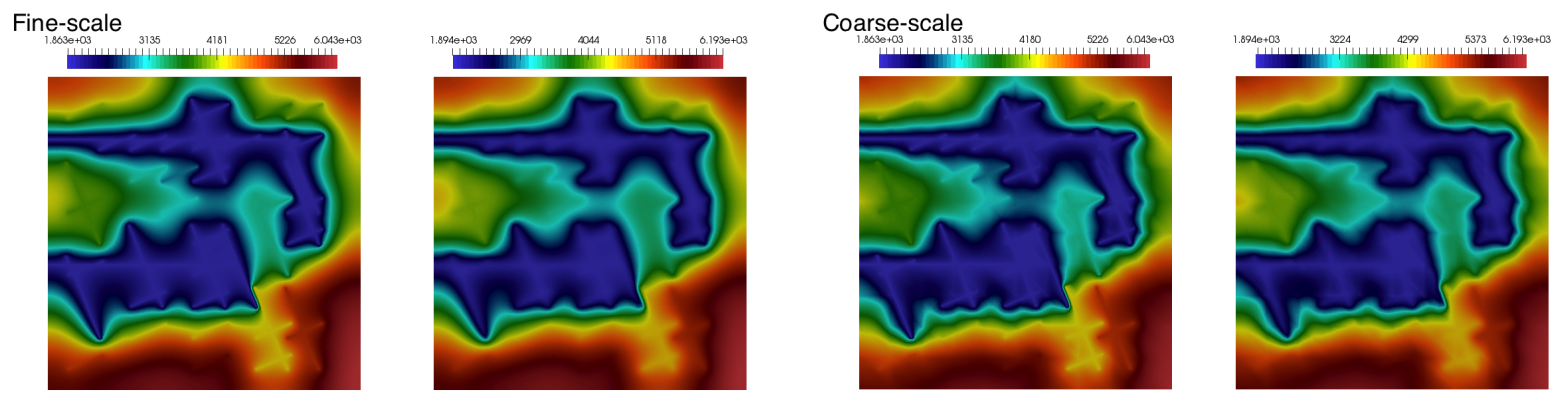

Figure 9: Dual-continuum background for shale gas transport. Left: Fine-scale solution $D O F=18064$. Right: Coarse-scale solution $D O F=938$ using simplified basis functions.

\section{Conclusions}

In this paper, our goals are: (1) to investigate the GMsFEM for fractured media; (2) to study the relation between the GMsFEM and the multi-continuum approaches; (3) to develop a coupled GMsFEM and multi-continuum approaches for highly heterogeneous fractured media. First, we show that GMsFEM basis functions represent each continuum and these multiscale basis functions correspond to the eigenvectors associated with very small eigenvalues. We propose simplified basis functions when fracture geometries are simple. Multiscale basis functions contain the spatial information representing the interaction between the matrix and the fractures. Numerical results show that the GMsFEM can provide an accurate solution if we include multiscale basis functions corresponding to very small eigenvalues. The latter represents the number of the continua in each coarse block. In the second part of the paper, we develop a coupled GMsFEM and multi-continuum approaches. In this case, fractures at the fine-subgrid are represented by a multi-continuum approach. As a result, the GMsFEM is needed for a system of equations. In this case, we use un-coupled and coupled basis functions. In the latter, the multiscale basis functions are constructed for subgrid multi-continuum media in a coupled fashion. We present numerical results, where we compute the parameters of the multi-continua from a subgrid problem. Our numerical 
results show that the GMsFEM is able to give solutions with good accuracy. The accuracy is better when using coupled basis functions.

\section{A Convergence analysis}

In this appendix, we present the convergence analysis of our schemes. We will consider both the un-coupled multiscale basis functions and the coupled multiscale basis functions as well as an abstract formulation to be defined in the following. Note that the abstract formulation can be applied to the practical cases presented in this paper. We consider the $N$-continuum problem: find $u=\left(u_{1}, u_{2}, \cdots u_{N}\right)$ such that $u_{i}(t, \cdot) \in H^{1}(\Omega), i=1, \cdots, N$, and

$$
\sum_{i} c_{i}\left(\frac{\partial u_{i}}{\partial t}, v_{i}\right)=-\sum_{i} a_{i}\left(u_{i}, v_{i}\right)+q(u, v)+(f, v), \quad t \in(0, T)
$$

for all test functions $v=\left(v_{1}, v_{2}, \cdots, v_{N}\right)$ with $v_{i}(t, \cdot) \in H_{0}^{1}(\Omega)$, where

$$
\begin{aligned}
c_{i}(u, v) & =\int_{D_{m}} c_{i} u v d x+\sum_{j} \int_{D_{f, j}} c_{j, i} u v d x, \\
q(u, v) & =\sum_{j} \sum_{i \neq j} Q_{i} \int_{D}\left(u_{i}-u_{j}\right) v_{j} d x, \\
a_{i}(u, v) & =\int_{D_{m}} \kappa_{i} \nabla u \cdot \nabla v d x+\sum_{j} \int_{D_{f, j}} \kappa_{j, i} \nabla_{f} u \cdot \nabla_{f} v d x .
\end{aligned}
$$

Note that all the summations are summing over all continua, that is, they are summing over $i, j=1,2, \cdots, N$. Next we define two global bilinear operators $c(\cdot, \cdot)$ and $a(\cdot, \cdot)$ by

$$
c(u, v)=\sum_{i} c_{i}\left(u_{i}, v_{i}\right), \quad a(u, v)=\sum_{i} a_{i}\left(u_{i}, v_{i}\right)
$$

Clearly, we have $q(u, v)=q(v, u)$ and $q(u, u) \leq 0$ for all $u(t, \cdot), v(t, \cdot) \in\left[H^{1}(\Omega)\right]^{N}$. Equation (12) defines our multi-continuum problem.

We next define the operator $a_{i}^{(j)}(\cdot, \cdot)$ by

$$
a_{i}^{(j)}(u, v)=\left(\int_{\omega_{j}} \kappa_{i} \nabla u \cdot \nabla v d x+\sum_{l} \int_{D_{f, l} \cap \omega_{j}} \kappa_{l, i} \nabla_{f} u \cdot \nabla_{f} v d x\right)
$$

for all $u(t, \cdot), v(t, \cdot) \in H_{0}^{1}\left(\omega_{j}\right)$. This operator corresponds to the contribution of $a_{i}(u, v)$ in the coarse region $\omega_{j}$. We also define the corresponding global operator

$$
a^{(j)}(u, v)=\sum_{i} a_{i}^{(j)}\left(u_{i}, v_{i}\right)
$$


Finally, we define two bilinear operators $a_{Q}^{(j)}(\cdot, \cdot)$ and $a_{Q}(\cdot, \cdot)$ by

$$
a_{Q}^{(j)}(u, v)=a^{(j)}(u, v)-q(u, v), \quad a_{Q}(u, v)=a(u, v)-q(u, v)
$$

for all $u(t, \cdot), v(t, \cdot) \in H_{0}^{1}\left(\omega_{j}\right)$.

In the following, we will present the definitions of the un-coupled multiscale basis functions and the coupled multiscale basis functions. For each case, we follow the general procedure to first construct a local snapshot space for each coarse region $\omega_{j}$, and then construct an offline space (consisting of multiscale basis functions) using a suitable spectral problem defined on the snapshot space. Note that the snapshot functions and the basis functions are independent of time.

\section{Coupled GMsFEM (snapshot space)}

For each coarse region $\omega_{j}$, we obtain the $k$-th snapshot function by solving the following local problem: find $\psi_{k}^{(j) \text {,snap }} \in\left[V_{h}\left(\omega_{j}\right)\right]^{N}$ such that

$$
a^{(j)}\left(\psi_{k}^{(j), \text { snap }}, v\right)-q\left(\psi_{k}^{(j), \text { snap }}, v\right)=0, \quad \forall v \in\left[V_{h, 0}\left(\omega_{j}\right)\right]^{N},
$$

with the boundary condition $\quad \psi_{k}^{(j), \text { snap }}=\delta_{k}$, on $\partial \omega_{j}$,

where $V_{h}\left(\omega_{j}\right)$ is a fine-scale space and $V_{h, 0}\left(\omega_{j}\right)$ is the subspace of $V_{h}\left(\omega_{j}\right)$ containing functions with zero trace on the boundary of $\omega_{j}$. In the above definition, the discrete delta function $\delta_{k}$ is defined as $\delta_{k}=\left(\delta_{k, 1}, \delta_{k, 2}, \cdots, \delta_{k, N}\right)$ and each $\delta_{k, i}$ is the discrete delta function such that $\delta_{k, i}=1$ at the fine-grid node $x_{k} \in \partial \omega_{j}$ and $\delta_{k, i}=0$ at all other fine-grid nodes on $\partial \omega_{j}$. Using the above snapshot functions, we can define the local snapshot space by

$$
V_{\text {snap }}\left(\omega_{j}\right)=\operatorname{span}\left\{\psi_{k}^{(j), \text { snap }}: \forall k\right\}
$$

\section{Coupled GMsFEM (offline space)}

We will construct the offline space in this section. The offline space is spanned by all multiscale basis functions. To find the multiscale basis functions, we use a local spectral problem defined in the snapshot space. More precisely, for each coarse region $\omega_{j}$, we consider the following local eigenvalue problem: find the $k$-th eigenfunction $\phi_{k}^{(j)} \in V_{\text {snap }}\left(\omega_{j}\right)$ and the $k$-th eigenvalue $\lambda_{k}^{(j)}$ such that

$$
a_{Q}^{(j)}\left(\phi_{k}^{(j)}, v\right)=\lambda_{i}^{(j)} s^{(j)}\left(\phi_{k}^{(j)}, v\right), \quad \forall v \in V_{\text {snap }}\left(\omega_{j}\right)
$$

where the bilinear form $s^{(j)}$ is defined as

$$
s^{(j)}(u, v)=\sum_{i}\left(\int_{\omega_{j}} \kappa_{i}\left|\nabla \chi_{j}\right|^{2} u v d x+\sum_{l} \int_{D_{f, l}} \kappa_{l, i}\left|\nabla_{f} \chi_{j}\right|^{2} u v d x\right)
$$


and the eigenvalues are arranged in ascending order. Using the eigenfunction $\phi_{k}^{(j)}$, we can define the $k$-th multiscale basis function by $\hat{\phi}_{k}^{(j)}=\chi_{j} \phi_{k}^{(j)}$, where $\left\{\chi_{j}\right\}$ is a set of partition of unity functions for the coarse-grid partition of the domain $\Omega$. Finally, the local offline space is defined by $V_{H}\left(\omega_{j}\right)=\operatorname{span}\left\{\hat{\phi}_{i}^{(j)} \mid i \leq L_{j}\right\}$, which is formed by using the first $L_{j}$ eigenfunctions. In addition, the global offline space, $V_{H}$, is defined by $V_{H}=\sum_{j} V_{H}\left(\omega_{j}\right)$.

\section{Un-Coupled GMsFEM (snapshot space)}

Now, we will present the construction of the basis for the un-coupled case. We first consider the construction of the snapshot space. For each coarse region $\omega_{j}$ and for each continuum

$i$, we obtain the $k$-th snapshot function by solving the problem: find $\psi_{k, i}^{(j) \text { snap }} \in V_{h}\left(\omega_{j}\right)$ such that

$$
\begin{aligned}
a_{i}^{(j)}\left(\psi_{k, i}^{(j), \text { snap }}, v\right) & =0, \quad \forall v \in V_{h, 0}\left(\omega_{j}\right), \\
\text { with the boundary condition } \quad \psi_{k, i}^{(j), \text { snap }} & =\delta_{k, i}, \quad \text { on } \partial \omega_{j} .
\end{aligned}
$$

Then, the local snapshot space for the coarse region $\omega_{j}$ and for the $i$-th continuum is defined by

$$
V_{\text {snap }}^{(i)}\left(\omega_{j}\right)=\operatorname{span}\left\{\psi_{k, i}^{(j), \text { snap }}: \forall k\right\}, \quad i=1,2, \cdots, N .
$$

\section{Un-Coupled GMsFEM (offline space)}

We will construct multiscale basis functions for each coarse region $\omega_{j}$ and for each continuum $i$. To do so, we consider the following local eigenvalue problem: find the $k$-th eigenfunction $\phi_{k, i}^{(j)} \in V_{\text {snap }}^{(i)}\left(\omega_{j}\right)$ and the $k$-th eigenvalue $\lambda_{k, i}^{(j)}$ such that

$$
a_{i}^{(j)}\left(\phi_{k, i}^{(j)}, v\right)=\lambda_{k, i}^{(j)} s_{i}^{(j)}\left(\phi_{k, i}^{(j)}, v\right), \quad \forall v \in V_{\text {snap }}^{(i)}\left(\omega_{j}\right)
$$

where

$$
s_{i}^{(j)}(u, v)=\int_{\omega_{j}} \kappa_{i}\left|\nabla \chi_{j}\right|^{2} u v d x+\sum_{l} \int_{D_{f, l}} \kappa_{l, i}\left|\nabla_{f} \chi_{j}\right|^{2} u v d x .
$$

We assume that the eigenvalues are arranged in ascending order. Using the above eigenfunctions, we can define the $k$-th multiscale basis function by $\hat{\phi}_{k, i}^{(j)}=\chi_{j} \phi_{k, i}^{(j)}$. To define the offline space for the $i$-th continuum and for the coarse region $\omega_{j}$, we take the first $L_{j}$ eigenfunctions and define $V_{H}^{(i)}\left(\omega_{j}\right)=\operatorname{span}\left\{\hat{\phi}_{k, i}^{(j)} \mid k \leq L_{j}\right\}$. Note that $L_{j}$ can depend on $i$, but we omit this index to simplify the notation. Then the global offline space for the $i$-th continuum is given by $V_{H}^{(i)}=\sum_{j} V_{H}^{(i)}\left(\omega_{j}\right)$. Finally, the offline space, $V_{H}$, is defined by $V_{H}=V_{H}^{1} \times V_{H}^{2} \times \cdots \times V_{H}^{N}$.

\section{Analysis}

Now we are ready to present the analysis. We will first prove the following best approximation estimates (see Lemma 1 and Lemma 2). We will compare the difference between the reference 
solution $u$, defined by $(12)$, and the multiscale solution $u_{m s} \in V_{H}$ defined by

$$
\sum_{i} c_{i}\left(\frac{\partial u_{m s, i}}{\partial t}, v_{i}\right)=-\sum_{i} a_{i}\left(u_{m s, i}, v_{i}\right)+q\left(u_{m s}, v\right)+(f, v), \forall v \in V_{H}, t \in(0, T) .
$$

We also define the following norms

$$
\|u\|_{c}^{2}=c(u, u), \quad\|u\|_{a}^{2}=a(u, u), \quad\|u\|_{a_{Q}}^{2}=a_{Q}(u, u) .
$$

Lemma 1. Let $u$ be the reference solution defined in (12) and $u_{m s}$ be the multiscale numerical solution defined in (13). We have

$$
\begin{aligned}
& \left\|u(t, \cdot)-u_{m s}(t, \cdot)\right\|_{c}^{2}+\int_{0}^{T}\left\|u-u_{m s}\right\|_{a_{Q}}^{2} d t \\
\leq & C \inf _{w \in V_{H}}\left(\int_{0}^{T}\left\|\frac{\partial(w-u)}{\partial t}\right\|_{c}^{2} d t+\int_{0}^{T}\|w-u\|_{a_{Q}}^{2} d t+\|w(0, \cdot)-u(0, \cdot)\|_{c}^{2}\right) .
\end{aligned}
$$

Proof. We write $u_{m s}=\left(u_{m s, 1}, \cdots, u_{m s, N}\right)$, where $u_{m s, i}$ is the component for the $i$-th continuum. Using (12) and (13), we have

$$
c\left(\frac{\partial\left(u-u_{m s}\right)}{\partial t}, v\right)+\sum_{i} a_{i}\left(u_{i}-u_{m s, i}, v\right)-q\left(u-u_{m s}, v\right)=0, \quad \forall v \in V_{H}, t \in(0, T) .
$$

Let $w \in V_{H}$ and $v=w-u_{m s}$ in the above equation, we obtain

$$
\begin{aligned}
& c\left(\frac{\partial\left(w-u_{m s}\right)}{\partial t}, w-u_{m s}\right)+\sum_{i} a_{i}\left(w_{i}-u_{m s, i}, w_{i}-u_{m s, i}\right)-q\left(w-u_{m s}, w-u_{m s}\right) \\
= & c\left(\frac{\partial(w-u)}{\partial t}, w-u_{m s}\right)+\sum_{i} a_{i}\left(w-u_{i}, w-u_{m s, i}\right)-q\left(w-u, w-u_{m s}\right) \\
\leq & \left\|\frac{\partial(w-u)}{\partial t}\right\|_{c}\left\|w-u_{m s}\right\|_{c}+\|w-u\|_{a_{Q}}\left\|w-u_{m s}\right\|_{a_{Q}} .
\end{aligned}
$$

Therefore, integrating the above in time, we obtain (14).

In the next lemma, we prove a similar result as (14) by assuming an additional condition on $q$, namely,

$$
-q(v, v) \leq D\|v\|_{a}^{2}, \quad \forall \quad v \in\left[H^{1}(\Omega)\right]^{N} .
$$

Lemma 2. Assume that $-q(v, v) \leq D\|v\|_{a}^{2}, \forall v \in\left[H^{1}(\Omega)\right]^{N}$. For the same $u$ and $u_{m s}$ as in Lemma 1, we have

$$
\begin{aligned}
& \left\|u(t, \cdot)-u_{m s}(t, \cdot)\right\|_{c}^{2}+\int_{0}^{T}\left\|u-u_{m s}\right\|_{a}^{2} d t \\
\leq & C \inf _{w \in V_{H}}\left(\int_{0}^{T}\left\|\frac{\partial(w-u)}{\partial t}\right\|_{c}^{2} d t+(D+1) \int_{0}^{T}\|w-u\|_{a}^{2} d t+\|w(0, \cdot)-u(0, \cdot)\|_{c}^{2}\right) .
\end{aligned}
$$


Proof. Since $\int_{0}^{T}\left\|u-u_{m s}\right\|_{a}^{2} d t \leq \int_{0}^{T}\left\|u-u_{m s}\right\|_{a_{Q}}^{2} d t$ and $-q(v, v) \leq D\|v\|_{a}^{2}$, we have

$$
\begin{aligned}
& \left\|u(t, \cdot)-u_{m s}(t, \cdot)\right\|_{c}^{2}+\int_{0}^{T}\left\|u-u_{m s}\right\|_{a}^{2} d t \\
\leq & \left\|u(t, \cdot)-u_{m s}(t, \cdot)\right\|_{c}^{2}+\int_{0}^{T}\left\|u-u_{m s}\right\|_{a_{Q}}^{2} d t \\
\leq & C \inf _{w \in V_{H}}\left(\int_{0}^{T}\left\|\frac{\partial(w-u)}{\partial t}\right\|_{c}^{2} d t+\int_{0}^{T}\|w-u\|_{a}^{2}-q(w-u, w-u) d t+\|w(0, \cdot)-u(0, \cdot)\|_{c}^{2}\right) \\
\leq & C \inf _{w \in V_{H}}\left(\int_{0}^{T}\left\|\frac{\partial(w-u)}{\partial t}\right\|_{c}^{2} d t+(D+1) \int_{0}^{T}\|w-u\|_{a}^{2} d t+\|w(0, \cdot)-u(0, \cdot)\|_{c}^{2}\right) .
\end{aligned}
$$

This completes the proof.

We will use the above two lemmas to prove the convergence of our scheme. In particular, we need to find a suitable function $w \in V_{H}$ and estimate the difference $w-u$ in various norms. The following is our strategy. We define the snapshot projection $u_{\text {snap }} \in V_{\text {snap }}$ by

$$
u_{\text {snap }}=\sum_{j} \chi_{j} u_{\text {snap }}^{(j)}, \quad \text { with }\left.u_{\text {snap }}^{(j)}\right|_{\partial \omega_{j}}=\left.u\right|_{\partial \omega_{j}},
$$

where $V_{\text {snap }}$ is the snapshot space obtained by collecting all snapshot functions. We note that, since the snapshot functions for each coarse region $\omega_{j}$ take all possible values on $\partial \omega_{j}$, the problem in (16) is well-defined. Since $w-u=w-u_{\text {snap }}+u_{\text {snap }}-u$, it suffices to estimates the two terms $w-u_{\text {snap }}$ and $u_{\text {snap }}-u$. Note that the term $u_{\text {snap }}-u$ corresponds to an irreducible error of our scheme, since this error cannot be improved by using our scheme. We assume that this irreducible error is small by using a large set of snapshot functions. Based on this argument, it suffices to estimate $w-u_{\text {snap }}$ by choosing an appropriate function $w \in V_{H}$.

Note that $u_{\text {snap }}$ is in the snapshot space, which means that we can represent $u$ as a linear combination of all multiscale basis functions. To define $w \in V_{H}$, we will take $w$ as the projection of $u_{\text {snap }}$ in the offline space. More precisely, we use the following construction. First, for the case of un-coupled basis functions, we can represent

$$
u_{\text {snap }}=\left(u_{\text {snap }, 1}, u_{\text {snap }, 2}, \cdots, u_{\text {snap }, N}\right), \quad u_{\text {snap }, i}=\sum_{j} \sum_{k} c_{k, i}^{(j)}(t) \chi_{j}(x) \phi_{k, i}^{(j)}(x) .
$$

Then the projection $w$ of $u$ in the offline space is defined as

$$
w=\left(w_{1}, w_{2}, \cdots, w_{N}\right), \quad w_{i}=\sum_{j} \sum_{k \leq L_{j}} c_{k, i}^{(j)}(t) \chi_{j}(x) \phi_{k, i}^{(j)}(x) .
$$

Second, for the case of coupled basis functions, we can represent

$$
u_{\text {snap }}=\sum_{j} \sum_{k} c_{k}^{(j)}(t) \chi_{j}(x) \phi_{k}^{(j)}(x) .
$$


Then the projection $w$ of $u_{\text {snap }}$ in the offline space is defined as

$$
w=\sum_{j} \sum_{k \leq L_{j}} c_{k}^{(j)}(t) \chi_{j}(x) \phi_{k}^{(j)}(x) .
$$

Next, we will state and prove the main results (Theorem 1 and Theorem 2) of this appendix. As we will see, Theorem 1 and Theorem 2 follow from Lemmas 3, 5, and 6 .

Theorem 1. For the un-coupled GMsFEM, let $u$ and $u_{\text {snap }}$ be the reference solution and snapshot projection in (12) and (13) and let $w \in V_{H}$ be the projection of $u_{\text {snap }}$ defined in (18). We assume (15). Then we have

$$
\begin{aligned}
& \int_{0}^{T}\left\|\frac{\partial\left(w-u_{\text {snap }}\right)}{\partial t}\right\|_{c}^{2} d t+\int_{0}^{T}\left\|w-u_{\text {snap }}\right\|_{a}^{2} d t+\left\|w(0, \cdot)-u_{\text {snap }}(0, \cdot)\right\|_{c}^{2} \\
\leq & \frac{C}{\Lambda_{1}}\left(\int_{0}^{T}\left\|\frac{\partial u}{\partial t}\right\|_{a}^{2} d t+\int_{0}^{T}\|u\|_{a}^{2} d t+\|u(0, \cdot)\|_{a}^{2}\right),
\end{aligned}
$$

where $\Lambda_{1}=\min _{j, i}\left\{\lambda_{L_{j}+1, i}^{(j)}\right\}$.

Theorem 2. For the coupled GMsFEM, let $u$ and $u_{\text {snap }}$ be the reference solution and snapshot projection in (12) and (13) and let $w \in V_{H}$ be the projection of $u_{\text {snap }}$ defined in (20). Then we have

$$
\begin{aligned}
& \int_{0}^{T}\left\|\frac{\partial\left(w-u_{\text {snap }}\right)}{\partial t}\right\|_{c}^{2} d t+\int_{0}^{T}\left\|w-u_{\text {snap }}\right\|_{a_{Q}}^{2} d t+\left\|w(0, \cdot)-u_{\text {snap }}(0, \cdot)\right\|_{c}^{2} \\
\leq & \frac{C^{2}}{\Lambda_{2}}\left(\int_{0}^{T}\left\|\frac{\partial u}{\partial t}\right\|_{a_{Q}}^{2} d t+\int_{0}^{T}\|u\|_{a_{Q}}^{2} d t+\|u(0, \cdot)\|_{a_{Q}}^{2}\right),
\end{aligned}
$$

where $\Lambda_{2}=\min _{j}\left\{\lambda_{L_{j}+1}^{(j)}\right\}$.

We will proof the above two theorems by estimating $\int_{0}^{T}\left\|\frac{\partial\left(w-u_{\text {snap }}\right)}{\partial t}\right\|_{c}^{2} d t, \int_{0}^{T} \| w-$ $u_{\text {snap }}\left\|_{a}^{2}, \int_{0}^{T}\right\| w-u_{\text {snap }} \|_{a_{Q}}^{2} d t$, and $\left\|w(0, \cdot)-u_{\text {snap }}(0, \cdot)\right\|_{c}^{2}$ separately in the following lemmas. Unless otherwise specified, the constant $C$ is independent of any scales and continuum.

Lemma 3. Let $u, u_{\text {snap }}$, and $w$ be defined as in Theorems 1 and 2. For the un-coupled basis functions, we have

$$
\int_{0}^{T}\left\|\frac{\partial\left(w-u_{\text {snap }}\right)}{\partial t}\right\|_{c}^{2} d t \leq \frac{C E}{\Lambda_{1}}\left\|\frac{\partial u}{\partial t}\right\|_{a}^{2} d t .
$$

For the coupled basis functions, we have

$$
\int_{0}^{T}\left\|\frac{\partial\left(w-u_{\text {snap }}\right)}{\partial t}\right\|_{c}^{2} \leq \frac{C E}{\Lambda_{2}}\left\|\frac{\partial u}{\partial t}\right\|_{a_{Q}}^{2},
$$

where $E=\max _{i, j, l}\left\{\frac{c_{i} \chi_{j}^{2}}{\kappa_{i}\left|\nabla \chi_{j}\right|^{2}}, \frac{c_{l, i} \chi_{j}^{2}}{\kappa_{l, i}\left|\nabla_{f} \chi_{j}\right|^{2}}\right\}$. 
Proof. We will present the proof for the case of un-coupled basis functions. First, note that

$$
\begin{aligned}
\left\|\left(u_{\text {snap }}\right)_{t}-w_{t}\right\|_{c}^{2} \leq & \sum_{i}\left\|\sum_{j}\left(\chi_{j} \frac{\partial u_{\text {snap }, i}^{(j)}}{\partial t}-\sum_{k \leq L_{j}} \frac{\partial c_{k, i}^{(j)}}{\partial t} \chi_{j} \phi_{k, i}^{(j)}\right)\right\|_{c}^{2} \\
\leq & D \sum_{i} \sum_{j} \int_{\omega_{j}} \frac{c_{i} \chi_{j}^{2}}{\kappa_{i}\left|\nabla \chi_{j}\right|^{2}} \kappa_{i}\left|\nabla \chi_{j}\right|^{2}\left(\frac{\partial u_{s n a p, i}^{(j)}}{\partial t}-\sum_{k \leq L_{j}} \frac{\partial c_{k, i}^{(j)}}{\partial t} \phi_{k, i}^{(j)}\right)^{2} d x \\
& +\sum_{i, j, l} \int_{D_{f, l} \cap \omega_{j}} c_{l, i} \frac{\chi_{j}^{2}}{\kappa_{l, i}\left|\nabla_{f} \chi_{j}\right|^{2}} \kappa_{l, i}\left|\nabla_{f} \chi_{j}\right|^{2}\left(\frac{\partial u_{\text {snap }, i}^{(j)}}{\partial t}-\sum_{k \leq L_{j}} \frac{\partial c_{k, i}^{(j)}}{\partial t} \phi_{k, i}^{(j)}\right)^{2} d x \\
\leq & D E \sum_{i} \sum_{j} s_{i}^{(j)}\left(\sum_{k>L_{j}} \frac{\partial c_{k, i}^{(j)}(t)}{\partial t} \phi_{k, i}^{(j)}, \sum_{k>L_{j}} \frac{\partial c_{k, i}^{(j)}(t)}{\partial t} \phi_{k, i}^{(j)}\right)
\end{aligned}
$$

with $D=\max _{K \in \mathcal{T}^{H}}\left\{D_{K}\right\}$ where $D_{K}$ is the number of coarse neighborhoods intersecting with $K$. By using the orthogonality of eigenfunctions, we have

$$
\begin{aligned}
s_{i}^{(j)}\left(\sum_{k>L_{j}} \frac{\partial c_{k, i}^{(j)}(t)}{\partial t} \phi_{k, i}^{(j)}, \sum_{k>L_{j}} \frac{\partial c_{k, i}^{(j)}(t)}{\partial t} \phi_{k, i}^{(j)}\right) & \leq \sum_{k>L_{j}} \frac{1}{\lambda_{k, i}^{(j)}}\left(\frac{\partial c_{k, i}^{(j)}(t)}{\partial t}\right)^{2} a_{i}^{(j)}\left(\phi_{k, i}^{(j)}, \phi_{k, i}^{(j)}\right) \\
& \leq \frac{1}{\lambda_{L_{j}+1, i}^{(j)}} \sum_{k}\left(\frac{\partial c_{k, i}^{(j)}(t)}{\partial t}\right)^{2} a_{i}^{(j)}\left(\phi_{k, i}^{(j)}, \phi_{k, i}^{(j)}\right) \\
& =\frac{1}{\lambda_{L_{j}+1, i}^{(j)}} a_{i}^{(j)}\left(\frac{\partial u_{\text {snap }, i}^{(j)}}{\partial t}, \frac{\partial u_{\text {snap }, i}^{(j)}}{\partial t}\right) .
\end{aligned}
$$

Since $u_{\text {snap }, i}^{(j)}$ is the $a_{i}^{(j)}$-harmonic expansion of $u_{i}$ in $\omega_{j}$, we have

$$
a_{i}^{(j)}\left(u_{\text {snap }}^{(j)}, u_{\text {snap }}^{(j)}\right) \leq a_{i}^{(j)}\left(u_{i}, u_{i}\right)
$$

and similarly

$$
a_{i}^{(j)}\left(\frac{\partial u_{\text {snap }, i}^{(j)}}{\partial t}, \frac{\partial u_{s n a p, i}^{(j)}}{\partial t}\right) \leq a_{i}^{(j)}\left(\frac{\partial u_{i}}{\partial t}, \frac{\partial u_{i}}{\partial t}\right) .
$$


Therefore, by summing over all $i, j$, we obtain

$$
\begin{aligned}
\sum_{i, j} s_{i}^{(j)}\left(\sum_{k>L_{j}} \frac{\partial c_{k, i}^{(j)}(t)}{\partial t} \phi_{k, i}^{(j)}, \sum_{k>L_{j}} \frac{\partial c_{k, i}^{(j)}(t)}{\partial t} \phi_{k, i}^{(j)}\right) & \leq \sum_{i, j} \frac{1}{\lambda_{L_{j}+1, i}^{(j)} a_{i}^{(j)}}\left(\frac{\partial u_{i}}{\partial t}, \frac{\partial u_{i}}{\partial t}\right) \\
& \leq \frac{1}{\min _{i, j}\left\{\lambda_{L_{j}+1, i}^{(j)}\right\}} \sum_{i, j} a_{i}^{(j)}\left(\frac{\partial u_{i}}{\partial t}, \frac{\partial u_{i}}{\partial t}\right) \\
& \leq \frac{D}{\min _{i, j}\left\{\lambda_{L_{j}+1, i}^{(j)}\right\}} a\left(\frac{\partial u}{\partial t}, \frac{\partial u}{\partial t}\right) .
\end{aligned}
$$

For the case of coupled basis functions, we have $s^{(j)}(\cdot, \cdot)=\sum_{i} s_{i}^{(j)}(\cdot, \cdot)$. By using the same arguments, we have

$$
\left\|\left(u_{\text {snap }}\right)_{t}-w_{t}\right\|_{c}^{2} \leq D E \sum_{j} s^{(j)}\left(\sum_{k>L_{j}} \frac{\partial c_{k}^{(j)}(t)}{\partial t} \phi_{k}^{(j)}, \sum_{k>L_{j}} \frac{\partial c_{k}^{(j)}(t)}{\partial t} \phi_{k}^{(j)}\right)
$$

and

$$
s^{(j)}\left(\sum_{k>L_{j}} \frac{\partial c_{k}^{(j)}(t)}{\partial t} \phi_{k}^{(j)}, \sum_{k>L_{j}} \frac{\partial c_{k}^{(j)}(t)}{\partial t} \phi_{k}^{(j)}\right) \leq \frac{1}{\lambda_{L_{j}+1}^{(j)}} a_{Q}^{(j)}\left(\frac{\partial u_{\text {snap }}^{(j)}}{\partial t}, \frac{\partial u_{\text {snap }}^{(j)}}{\partial t}\right) .
$$

Since $u_{\text {snap }}^{(j)}$ is the $a_{Q}^{(j)}$-harmonic expansion of $u_{i}$ in $\omega_{j}$, we have

$$
a_{Q}^{(j)}\left(u_{\text {snap }}^{(j)}, u_{\text {snap }}^{(j)}\right) \leq a_{Q}^{(j)}(u, u)
$$

and

$$
a_{Q}^{(j)}\left(\frac{\partial u_{\text {snap }}^{(j)}}{\partial t}, \frac{\partial u_{\text {snap }}^{(j)}}{\partial t}\right) \leq a_{Q}^{(j)}\left(\frac{\partial u}{\partial t}, \frac{\partial u}{\partial t}\right) .
$$

Therefore the proof is complete.

Before we estimate the terms $\int_{0}^{T}\left\|w-u_{\text {snap }}\right\|_{a}^{2} d t$ and $\int_{0}^{T}\left\|w-u_{\text {snap }}\right\|_{a_{Q}}^{2} d t$, we first prove the following lemma.

Lemma 4. For the case of coupled basis functions, if $u$ satisfies

$\sum_{i} \int_{\omega_{j}} \kappa_{i} \nabla u_{i} \cdot \nabla v_{i} d x+\sum_{l} \int_{D_{f, l} \cap \omega_{j}} \kappa_{l, i} \nabla_{f} u_{i} \cdot \nabla_{f} v_{i} d x-q(u, v)=\int_{\omega_{j}} f v d x, \quad \forall v \in\left[H_{0}^{1}\left(\omega_{j}\right)\right]^{N}$,

then we have

$$
\begin{aligned}
& \sum_{i} \int_{\omega_{j}} \kappa_{i} \chi_{j}^{2}\left|\nabla u_{i}\right|^{2} d x+\sum_{l} \int_{D_{f, l} \cap \omega_{j}} \kappa_{l, i} \chi_{j}^{2}\left|\nabla_{f_{j}} u\right|^{2}-q\left(\chi_{j} u, \chi_{j} v\right) d x \\
\leq & \left.C \sum_{i}\left(\int_{\omega} \frac{\chi_{j}^{4}}{\kappa_{i}\left|\nabla \chi_{j}\right|^{2}} f_{i}^{2} d x+\int_{\omega_{j}} \kappa_{i}\left|\nabla \chi_{j}\right|^{2} u^{2} d x\right]+\sum_{l} \int_{D_{f, l} \cap \omega_{j}} \kappa_{l, i}\left|\nabla_{f} \chi_{j}\right|^{2} u^{2} d x\right) .
\end{aligned}
$$


For the case of un-coupled basis functions, if u satisfies

$$
\int_{\omega_{j}} \kappa_{i} \nabla u \cdot \nabla v d x+\sum_{l} \int_{D_{f, l} \cap \omega_{j}} \kappa_{l, i} \nabla_{f} u \cdot \nabla_{f} v d x=\int_{\omega_{j}} f v d x, \quad \forall v \in H_{0}^{1}\left(\omega_{j}\right),
$$

then we have

$$
\begin{aligned}
& \sum_{i} \int_{\omega_{j}} \kappa_{i} \chi_{j}^{2}\left|\nabla u_{i}\right|^{2} d x+\sum_{l} \int_{D_{f, l} \cap \omega_{j}} \kappa_{l, i} \chi_{j}^{2}\left|\nabla_{f} u\right|^{2} d x \\
\leq & C \sum_{i}\left(\int \frac{\chi_{j}^{4}}{\kappa_{i}\left|\nabla \chi_{j}\right|^{2}} f_{i}^{2} d x+\int_{\omega_{j}} \kappa_{i}\left|\nabla \chi_{j}\right|^{2} u^{2} d x+\sum_{l} \int_{D_{f, l} \cap \omega_{j}} \kappa_{l, i}\left|\nabla_{f} \chi_{j}\right|^{2} u^{2} d x\right) .
\end{aligned}
$$

Proof. For the case of coupled basis functions, we take $v=\chi_{j}^{2} u$ and obtain

$$
\sum_{i} \int_{\omega_{j}} \kappa_{i} \nabla u_{i} \cdot \nabla\left(\chi_{j}^{2} u_{i}\right) d x+\sum_{l} \int_{D_{f, l} \cap \omega_{j}} \kappa_{l, i} \nabla_{f} u_{i} \cdot \nabla_{f}\left(\chi_{j}^{2} u_{i}\right) d x-q\left(\chi_{j} u, \chi_{j} u\right)=\int_{\omega_{j}} \chi_{j}^{2} f u d x .
$$

This implies

$$
\begin{aligned}
& \sum_{i}\left(\int_{\omega_{j}} \kappa_{i} \chi_{j}^{2}\left|\nabla u_{i}\right|^{2} d x+\sum_{l} \int_{D_{l, i} \cap \omega_{j}} \kappa_{l, i} \chi_{j}^{2}\left|\nabla_{f} u_{i}\right|^{2} d x-q\left(\chi_{j} u, \chi_{j} u\right)\right) \\
= & \int_{\omega_{j}} \chi_{j}^{2} f u d x-2 \sum_{i}\left(\int_{\omega_{j}} \kappa_{i} \chi_{j} u_{i} \nabla u_{i} \cdot \nabla \chi_{j} d x+\sum_{l} \int_{D_{f, l} \cap \omega_{j}} \kappa_{l, i} \chi_{j} u_{i} \nabla_{f} u_{i} \cdot \nabla_{f} \chi_{j} d x\right) \\
\leq & C \sum_{i}\left(\int \frac{\chi_{j}^{4}}{\kappa_{i}\left|\nabla \chi_{j}\right|^{2}} f_{i}^{2} d x+\int_{\omega_{j}} \kappa_{i}\left|\nabla \chi_{j}\right|^{2} u_{k}^{2} d x+\sum_{l} \int_{D_{f, l} \cap \omega_{j}} \kappa_{l, i}\left|\nabla \chi_{j}\right|^{2} u_{i}^{2} d x\right) .
\end{aligned}
$$

This completes the proof for the case of coupled basis functions. For the case of un-coupled basis functions, the proof is similar and is therefore omitted.

Lemma 5. Let $u, u_{\text {snap }}$ and $w$ be defined as in Theorem 1 and 2. For the case of un-coupled basis functions, we have

$$
\int_{0}^{T}\left\|w-u_{\text {snap }}\right\|_{a}^{2} d t \leq \frac{C}{\Lambda_{1}}\|u\|_{a}^{2}
$$

For the case of coupled basis functions, we have

$$
\int_{0}^{T}\left\|w-u_{\text {snap }}\right\|_{a_{Q}}^{2} d t \leq \frac{C}{\Lambda_{2}}\|u\|_{a_{Q}}^{2}
$$

where $\Lambda_{1}$ and $\Lambda_{2}$ are defined in Theorem 1 and 2 . 
Proof. We first define $e_{i}^{(j)}$ by

$$
e_{i}^{(j)}=\left\{\begin{aligned}
\sum_{k>L_{j}} \frac{\partial c_{k, i}^{(j)}(t)}{\partial t} \phi_{k, i}^{(j)}, & \text { for un-coupled basis functions } \\
\sum_{k>L_{j}} \frac{\partial c_{k}^{(j)}(t)}{\partial t} \phi_{k}^{(j)}, & \text { for coupled basis functions. }
\end{aligned}\right.
$$

Note that

$$
\begin{aligned}
& \left\|w-u_{\text {snap }}\right\|_{a}^{2} \\
= & \sum_{i}\left(\int_{\omega_{j}} \kappa_{i} \nabla \sum_{j} \chi_{j} e_{i}^{(j)} \cdot \nabla \sum_{j} \chi_{j} e_{i}^{(j)} d x+\sum_{l} \int_{D_{f, l} \cap \omega_{j}} \kappa_{l, i} \nabla_{f} \sum_{j} \chi_{j} e_{i}^{(j)} \cdot \nabla_{f} \sum_{j} \chi_{j} e_{i}^{(j)} d x\right) \\
\leq & D \sum_{i} \sum_{j}\left(\int_{\omega_{j}} \kappa_{i}\left|\nabla \chi_{j} e_{i}^{(j)}\right|^{2} d x+\sum_{l} \int_{D_{f, l} \cap \omega_{j}} \kappa_{l, i}\left|\nabla_{f} \chi_{j} e_{i}^{(j)}\right|^{2} d x\right) \\
\leq & C D \sum_{i, j}\left(\int_{\omega_{j}} \kappa_{i} \chi_{j}^{2}\left|\nabla e_{i}^{(j)}\right|^{2} d x+\sum_{l} \int_{D_{f, l} \cap \omega_{j}} \kappa_{l, i} \chi_{j}^{2}\left|\nabla_{f} e_{i}^{(j)}\right|^{2} d x+s_{i}^{(j)}\left(e_{i}^{(j)}, e_{i}^{(j)}\right)\right) .
\end{aligned}
$$

In addition, we have

$$
-q\left(\sum_{j} \chi_{j} e^{(j)}, \sum_{j} \chi_{j} e^{(j)}\right) \leq-D \sum_{j}\left(q\left(\chi_{j} e^{(j)}, \chi_{j} e^{(j)}\right)\right),
$$

where $D$ is defined in the proof of Lemma 3 .

For the case of coupled basis functions, using Lemma 4, we obtain

$$
\sum_{i} \int_{\omega_{j}} \kappa_{i}\left|\nabla \chi_{j} e_{i}^{(j)}\right|^{2} d x+\sum_{l} \int_{D_{f, l} \cap \omega_{j}} \kappa_{l, i}\left|\nabla_{f} \chi_{j} e_{i}^{(j)}\right|^{2} d x-q\left(\chi_{j} e^{(j)}, \chi_{j} e^{(j)}\right) \leq C s^{(j)}\left(e^{(j)}, e^{(j)}\right) .
$$

Therefore, we have

$$
\begin{aligned}
\left\|w-u_{\text {snap }}\right\|_{a_{Q}}^{2} & \leq\left\|w-u_{\text {snap }}\right\|_{a}^{2}-D \sum_{j}\left(q\left(\chi_{j} e^{(j)}, \chi_{j} e^{(j)}\right)\right) \\
& \leq C s^{(j)}\left(e^{(j)}, e^{(j)}\right) .
\end{aligned}
$$

For the case of un-coupled basis functions, using Lemma 4 again, we obtain

$$
\sum_{k} \int_{\omega_{j}} \kappa_{i}\left|\nabla \chi_{j} e_{k}^{(j)}\right|^{2} d x+\sum_{l} \int_{D_{f, l} \cap \omega_{j}} \kappa_{l, k}\left|\nabla_{f} \chi_{j} e_{k}^{(j)}\right|^{2} d x \leq C s^{(j)}\left(e^{(j)}, e^{(j)}\right) .
$$

Therefore, we have

$$
\left\|w-u_{\text {snap }}\right\|_{a}^{2} \leq C \sum_{i} s_{i}^{(j)}\left(e_{i}^{(j)}, e_{i}^{(j)}\right)
$$


Finally, by the definition of the eigen-projection, for the case of coupled basis functions, we have

$$
s^{(j)}\left(e^{(j)}, e^{(j)}\right) \leq \frac{1}{\lambda_{L_{j}+1}^{(j)}} a_{Q}^{(j)}\left(e^{(j)}, e^{(j)}\right) \leq \frac{1}{\lambda_{L_{j}+1}^{(j)}} a_{Q}^{(j)}\left(u_{\text {snap }}, u_{\text {snap }}\right) \leq \frac{1}{\lambda_{L_{j}+1}^{(j)}} a_{Q}^{(j)}(u, u)
$$

and, for the case of un-coupled basis functions, we have

$$
s_{i}^{(j)}\left(e_{i}^{(j)}, e_{i}^{(j)}\right) \leq \frac{1}{\lambda_{L_{j}+1, i}^{(j)}} a_{i}^{(j)}\left(e_{i}^{(j)}, e_{i}^{(j)}\right) \leq \frac{1}{\lambda_{L_{j}+1, i}^{(j)}} a_{i}^{(j)}\left(u_{\text {snap }, i}, u_{\text {snap }, i}\right) \leq \frac{1}{\lambda_{L_{j}+1, i}^{(j)}} a_{i}^{(j)}(u, u) .
$$

This completes the proof.

Finally, by using arguments similar as in the proof of Lemma 3 , we can prove the following lemma.

Lemma 6. Let $u, u_{\text {snap }}$, and $w$ be defined as in Theorem 1 and 2. For the case of un-coupled basis functions, we have

$$
\left\|w(0, \cdot)-u_{\text {snap }}(0, \cdot)\right\|_{c}^{2} \leq \frac{C E}{\Lambda_{1}}\|u(0, x)\|_{a}^{2}
$$

For the case of coupled basis functions, we have

$$
\left\|w(0, \cdot)-u_{\text {snap }}(0, \cdot)\right\|_{c}^{2} \leq \frac{C E}{\Lambda_{2}}\|u(0, x)\|_{a_{Q}}^{2}
$$

\section{References}

[1] I. Akkutlu, Y. Efendiev, And M. Vasilyeva, Multiscale model reduction for shale gas transport in fractured media, Computational Geosciences, (2015), pp. 1-21.

[2] I. Akkutlu, Y. Efendiev, M. Vasilyeva, And Y. Wang, Multiscale model reduction for shale gas transport in a coupled discrete fracture and dual-continuum media, Journal of Natural Gas Science and Engineering, (2016). submitted for Special Issue "Multiscale and Multiphysics Techniques and Their Applications in Unconventional Gas Reservoirs".

[3] I. Y. AkKutlu And E. FAthi, Multiscale gas transport in shales with local kerogen heterogeneities, SPE Journal, 17 (2012), pp. 1-002.

[4] T. Arbogast, J. Douglas, JR, And U. Hornung, Derivation of the double porosity model of single phase flow via homogenization theory, SIAM Journal on Mathematical Analysis, 21 (1990), pp. 823-836. 
[5] R. Baca, R. Arnett, And D. Langford, Modelling fluid flow in fractured-porous rock masses by finite-element techniques, International Journal for Numerical Methods in Fluids, 4 (1984), pp. 337-348.

[6] G. Barenblatt, I. P. Zheltov, and I. Kochina, Basic concepts in the theory of seepage of homogeneous liquids in fissured rocks [strata], Journal of applied mathematics and mechanics, 24 (1960), pp. 1286-1303.

[7] I. Bogdanov, V. Mourzenko, J.-F. Thovert, and P. Adler, Two-phase flow through fractured porous media, Physical Review E, 68 (2003), p. 026703.

[8] V. Calo, Y. Efendiev, J. Galvis, and G. Li, Randomized oversampling for generalized multiscale finite element methods, http://arxiv.org/pdf/1409.7114.pdf. to appear in SIAM MMS.

[9] E. Chung, Y. Efendiev, And T. Y. Hou, Adaptive multiscale model reduction with generalized multiscale finite element methods, Journal of Computational Physics, 320 (2016), pp. 69-95.

[10] E. T. Chung, Y. Efendiev, R. L. Gibson JR, And M. Vasilyeva, A generalized multiscale finite element method for elastic wave propagation in fractured media, GEMInternational Journal on Geomathematics, (2015), pp. 1-20.

[11] L. DuRlofsky, Numerical calculation of equivalent grid block permeability tensors for heterogeneous porous media, Water Resour. Res., 27 (1991), pp. 699-708.

[12] Y. Efendiev, J. Galvis, And T. Hou, Generalized multiscale finite element methods, Journal of Computational Physics, 251 (2013), pp. 116-135.

[13] Y. Efendiev, J. Galvis, And X. Wu, Multiscale finite element methods for highcontrast problems using local spectral basis functions, Journal of Computational Physics, 230 (2011), pp. 937-955.

[14] B. Eikemo, K.-A. Lie, G. T. Eigestad, And H. K. Dahle, Discontinuous galerkin methods for advective transport in single-continuum models of fractured media, Advances in water resources, 32 (2009), pp. 493-506.

[15] J. Erhel, J.-R. De Dreuzy, And B. Poirriez, Flow simulation in threedimensional discrete fracture networks, SIAM Journal on Scientific Computing, 31 (2009), pp. 2688-2705.

[16] S. Geiger-Boschung, S. K. MatthäI, J. Niessner, R. Helmig, et Al., Blackoil simulations for three-component, three-phase flow in fractured porous media, SPE journal, 14 (2009), pp. 338-354. 
[17] S. Granet, P. Fabrie, P. Lemonnier, And M. Quintard, A two-phase flow simulation of a fractured reservoir using a new fissure element method, Journal of Petroleum Science and Engineering, 32 (2001), pp. 35-52.

[18] H. Hoteit And A. FiroozABADI, Multicomponent fluid flow by discontinuous galerkin and mixed methods in unfractured and fractured media, Water Resources Research, 41 (2005).

[19] — An efficient numerical model for incompressible two-phase flow in fractured media, Advances in Water Resources, 31 (2008), pp. 891-905.

[20] R. Juanes, J. Samper, And J. Molinero, A general and efficient formulation of fractures and boundary conditions in the finite element method, International Journal for Numerical Methods in Engineering, 54 (2002), pp. 1751-1774.

[21] M. Karimi-FARd And L. Durlofsky, A general gridding, discretization, and coarsening methodology for modeling flow in porous formations with discrete geological features, Advances in Water Resources, 96 (2016), pp. 354-372.

[22] M. Karimi-Fard, L. Durlofsky, K. Aziz, et Al., An efficient discrete-fracture model applicable for general-purpose reservoir simulators, SPE Journal, 9 (2004), pp. 227-236.

[23] M. Karimi-Fard, A. Firoozabadi, et AL., Numerical simulation of water injection in fractured media using the discrete-fracture model and the galerkin method, SPE Reservoir Evaluation \& Engineering, 6 (2003), pp. 117-126.

[24] H. Kazemi, L. Merrill Jr, K. Porterfield, P. Zeman, et Al., Numerical simulation of water-oil flow in naturally fractured reservoirs, Society of Petroleum Engineers Journal, 16 (1976), pp. 317-326.

[25] J.-G. KIM AND M. D. DEO, Finite element, discrete-fracture model for multiphase flow in porous media, AIChE Journal, 46 (2000), pp. 1120-1130.

[26] J. MA, G. D. Couples, AND S. D. HARris, A mixed finite element technique based on implicit discretization of faults for permeability upscaling in fault damage zones, Water resources research, $42(2006)$.

[27] V. Martin, J. Jaffré, And J. E. Roberts, Modeling fractures and barriers as interfaces for flow in porous media, SIAM Journal on Scientific Computing, 26 (2005), pp. 1667-1691.

[28] S. K. Matthai, A. A. Mezentsev, M. Belayneh, et Al., Finite element-nodecentered finite-volume two-phase-flow experiments with fractured rock represented by unstructured hybrid-element meshes, SPE Reservoir Evaluation \& Engineering, 10 (2007), pp. $740-756$. 
[29] J. Monteagudo And A. Firoozabadi, Control-volume method for numerical simulation of two-phase immiscible flow in two- and three-dimensional discrete-fractured media, Water Resources Research, 40 (2004).

[30] H. Nick AND S. MATTHäI, Comparison of three fe-fv numerical schemes for singleand two-phase flow simulation of fractured porous media, Transport in porous media, 90 (2011), pp. 421-444.

[31] B. Noetinger, A quasi steady state method for solving transient darcy flow in complex $3 d$ fractured networks accounting for matrix to fracture flow, Journal of Computational Physics, 283 (2015), pp. 205-223.

[32] K. Pruess And T. NARAsimhan, On fluid reserves and the production of superheated steam from fractured, vapor-dominated geothermal reservoirs, Journal of Geophysical Research: Solid Earth, 87 (1982), pp. 9329-9339.

[33] V. Reichenberger, H. Jakobs, P. Bastian, and R. Helmig, A mixeddimensional finite volume method for two-phase flow in fractured porous media, Advances in Water Resources, 29 (2006), pp. 1020-1036.

[34] J. WARren, P. J. Root, ET AL., The behavior of naturally fractured reservoirs, Society of Petroleum Engineers Journal, 3 (1963), pp. 245-255.

[35] X. Wu, Y. Efendiev, And T. Hou, Analysis of upscaling absolute permeability, Discrete and Continuous Dynamical Systems, Series B., 2 (2002), pp. 158-204.

[36] Y.-S. Wu, K. Pruess, ET AL., A multiple-porosity method for simulation of naturally fractured petroleum reservoirs, SPE Reservoir Engineering, 3 (1988), pp. 327-336.

[37] B. Yan, Y. WAng, And J. E. KIllough, Beyond dual-porosity modeling for the simulation of complex flow mechanisms in shale reservoirs, Computational Geosciences, 20 (2016), pp. 69-91. 